\title{
Etude de la butée sur un écran de largeur limitée en sol frottant
}

\author{
Pierre Regenass* - Mohamed Meksaouine** \\ Richard Kastner** - Abdul-Hamid Soubra* — Yvan Bourdeau**
}

* Laboratoire d'Etudes et de Recherche en Génie Civil - ENSAIS

24, Boulevard de la Victoire

F-67084 Strasbourg cedex

/Pierre.Regenass, Ahamid.Soubra/@ensais.u-strasbg.fr

** URGC Géotechnique - INSA de Lyon

20, Avenue Albert Einstein

F-69621 Villeurbanne cedex

kastner@gcu-geot.insa-lyon.fr

RÉSUMÉ. De nombreux ouvrages mobilisent la résistance passive du sol de manière tridimensionnelle. L'objectif de cet article est de quantifier la butée des terres sur un écran de largeur limitée. Une campagne d'essais sur modèle réduit a permis de mettre en évidence différents modes de rupture suivant l'élancement de l'écran. Les schémas de rupture obtenus ont permis d'élaborer un modèle de calcul de la butée tridimensionnelle dans le cas des écrans peu élancés. L'outil théorique retenu est l'approche cinématique de l'analyse limite. Trois mécanismes de rupture de type translationnel sont envisagés. Les résultats obtenus ont permis de dégager le meilleur majorant de l'effort de butée.

ABSTRACT. This paper deals with the problem of the three dimensional passive earth pressures. First, the model tests carried out allows one to deduce the mode of failure of wall of limited breadth. Then, a theoretical study based on the kinematical approach of the limit analysis theory is investigated. Three translational kinematically admissible failure mechanisms are considered. The smallest upper-bound solutions of the three dimensional passive earth pressures are presented and discussed.

MOTS-CLÉS : butée - soutènement - analyse limite - sol frottant - effet tridimensionnel

KEY WORDS: passive pressure - retaining structure - limit analysis - cohesionless soil - threedimensional effect 


\section{Introduction}

De nombreux ouvrages, tels que les massifs de réaction, les corps d'ancrage ou les écrans de soutènement ayant une fiche discontinue (parois berlinoises, rideaux de palplanches à jambes de pantalon...) mobilisent la résistance passive du sol de manière tridimensionnelle. Si le problème de la butée bidimensionnelle provoquée par des écrans filants a fait l'objet de très nombreuses études tant expérimentales que théoriques, il n'en est pas de même de la butée tridimensionnelle provoq des écrans de largeur limitée. On dispose essentiellement de résultats d'essais sur modèles présentant une forte dispersion (cf. [HOR 67]). Pour le calcul, Blum [BLU 32] a présenté une extension à trois dimensions du schéma du coin de Coulomb alors que Horn propose une règle empirique déduite des expériences qu'il a analysées. Les limites de ces résultats nous ont conduits d'une part à effectuer des expérimentations sur modèles bidimensionnels et tridimensionnels, dont les conclusions sont rappelées ici, d'autre part à développer trois mécanismes de rupture dans le cadre de l'approche cinématique de la théorie de l'analyse limite.

\section{Etude expérimentale de la butée tridimensionnelle}

\subsection{Les objectifs et les conditions d'essais}

Horn [HOR 72] a présenté une synthèse d'une vingtaine de recherches expérimentales sur la butée tridimensionnelle. La plupart des essais sont effectués sur sable sec avec des écrans dont la hauteur ne dépasse pas $0,5 \mathrm{~m}$. Les modèles de Weissenbach [WEI 61] et Horn [HOR 67] échappent à cette règle, d'une part parce que la hauteur de l'écran peut y atteindre 2,5 m et d'autre part parce qu'ils ont fait l'objet de tests avec du sable humide et du gravier.

L'analyse de Horn fait apparaître de très fortes dispersions des résultats souvent difficiles à synthétiser en raison de la nature différente des matériaux, du manque de données sur leurs propriétés, et enfin des effets d'échelle liés aux différences de taille des modèles. Pour s'affranchir des effets dus à la taille des modèles, Belabdelouhab [BEL 88] et Meksaouine [MEK 93] ont utilisé le mêrne bâti d'essai et le même matériau que ceux utilisés par Bourdeau [BOU 77] pour l'étude de la butée bidimensionnelle. Ces essais conduisent ainsi à un ensemble cohérent de résultats allant des écrans filants à ceux d'élancement important. Nous analysons ici essentiellement les essais réalisés par Meksaouine dont le dispositif expérimental est décrit dans [MEK 93]. Lors de ces expérimentations, l'écran, dont la hauteur $h$ varie de 125 à $400 \mathrm{~mm}$ et sa largeur $b$ de 15 à $100 \mathrm{~mm}$, a été mis en translation avec une vitesse de $1 \mathrm{~mm} / \mathrm{min}$ environ, dans un massif de $1 \mathrm{~m}$ de haut, de $1,5 \mathrm{~m}$ de largeur et $1,5 \mathrm{~m}$ de longueur (figure 1). Son instrumentation permet de déterminer l'intensité de l'effort de butée, sa direction par rapport à la normale à l'écran, ainsi que sa position relative $X / h$ par rapport à la base de l'écran (figure 2). A la fin de chaque 
essai, un relevé de la surface débouchante du volume de sol en rupture est effectué. Cette surface est définie par ses dimensions frontales et latérales $L$ et $V$ (figure 1).

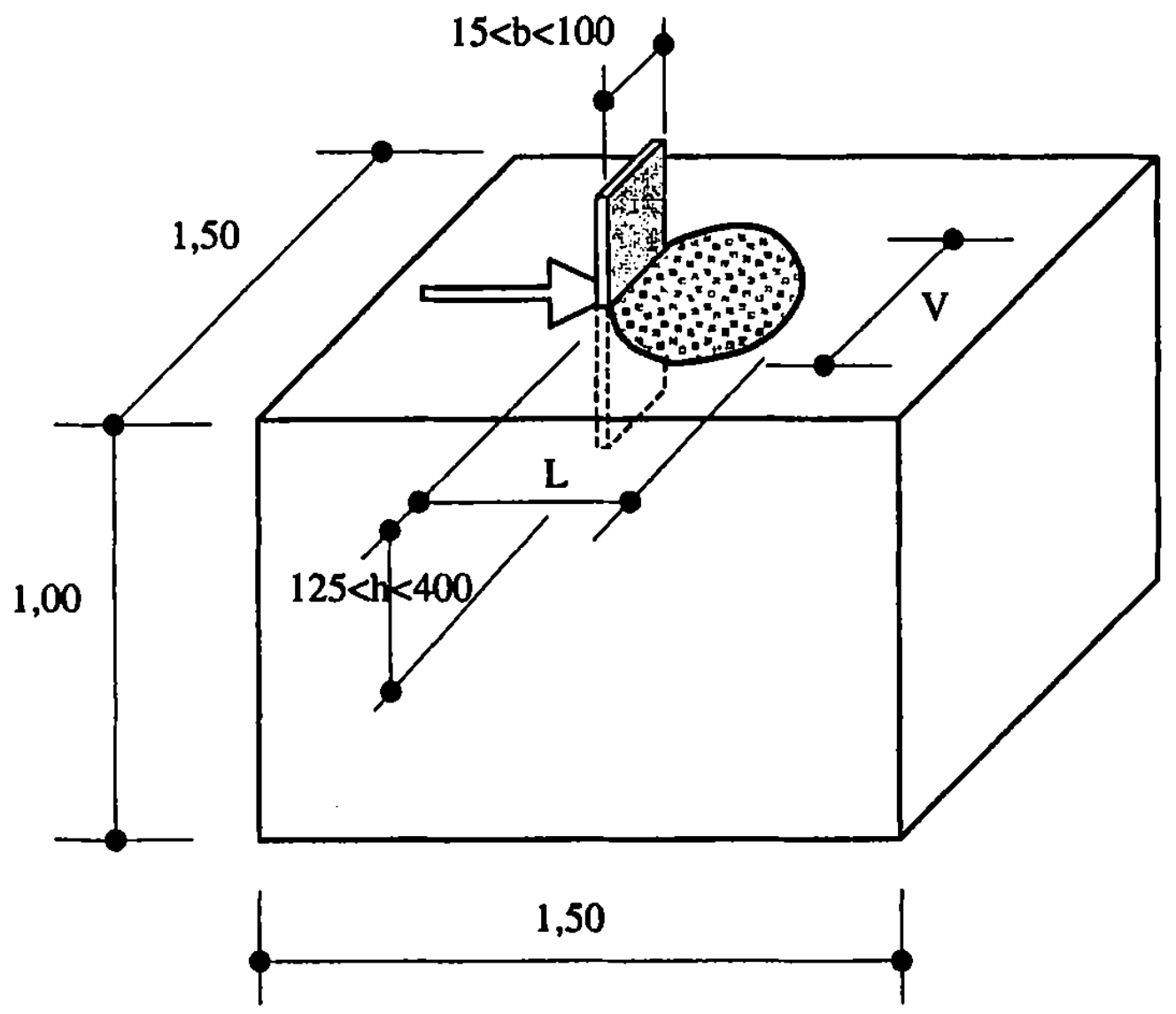

Figure 1. Schéma du dispositif expérimental. Experimental model

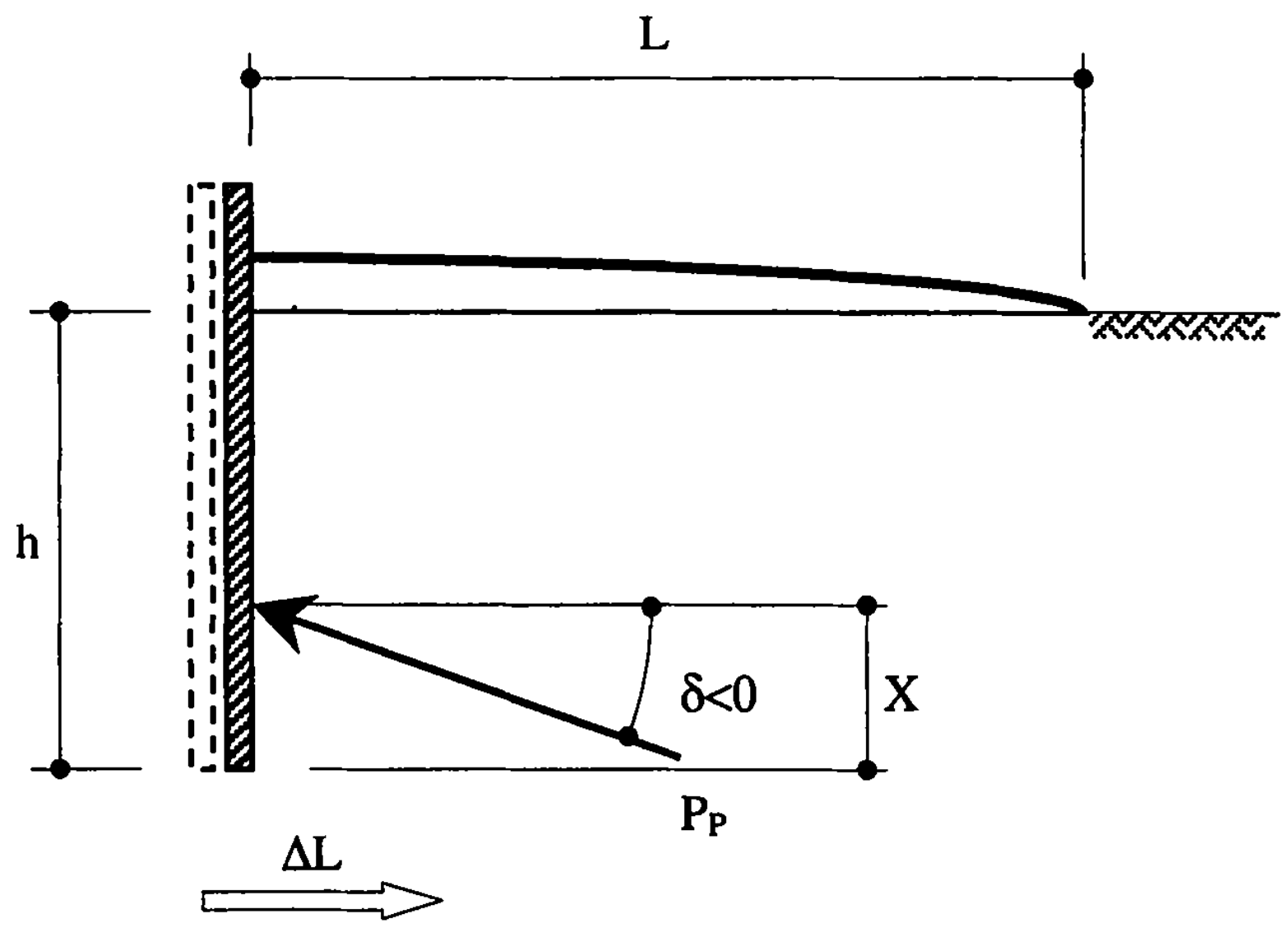

Figure 2. Prisme de rupture en butée. Passive failure wedge 
Pour tous les essais, le matériau utilisé est un sable d'Hostun $(D=1 \mathrm{~mm})$ obtenu par concassage dont les caractéristiques physiques et mécaniques sont données dans Bourdeau [BOU 77]. Dans les expérimentations de Meksaouine [MEK 93], ce sable est mis en place à une densité relative $I_{D}$ de $71 \%$ (soit $\gamma_{d}=15,6 \mathrm{kN} / \mathrm{m}^{3}$ ) qui correspond à un angle de frottement $\phi=34,5^{\circ}$.

\subsection{Prise en compte de l'effet de taille du modèle}

Les essais sur modèle réduit conventionnel non centrifugés ne permettent pas le respect des conditions de similitudes essentielles et peuvent être ainsi source d'importants effets d'échelle [COR 86]. Ainsi, l'étude de la portance de fondations superficielles ou de la butée sur modèle réduit conduit à des facteurs de portance ou des coefficients de butée adimensionnels variables avec la taille du modèle.

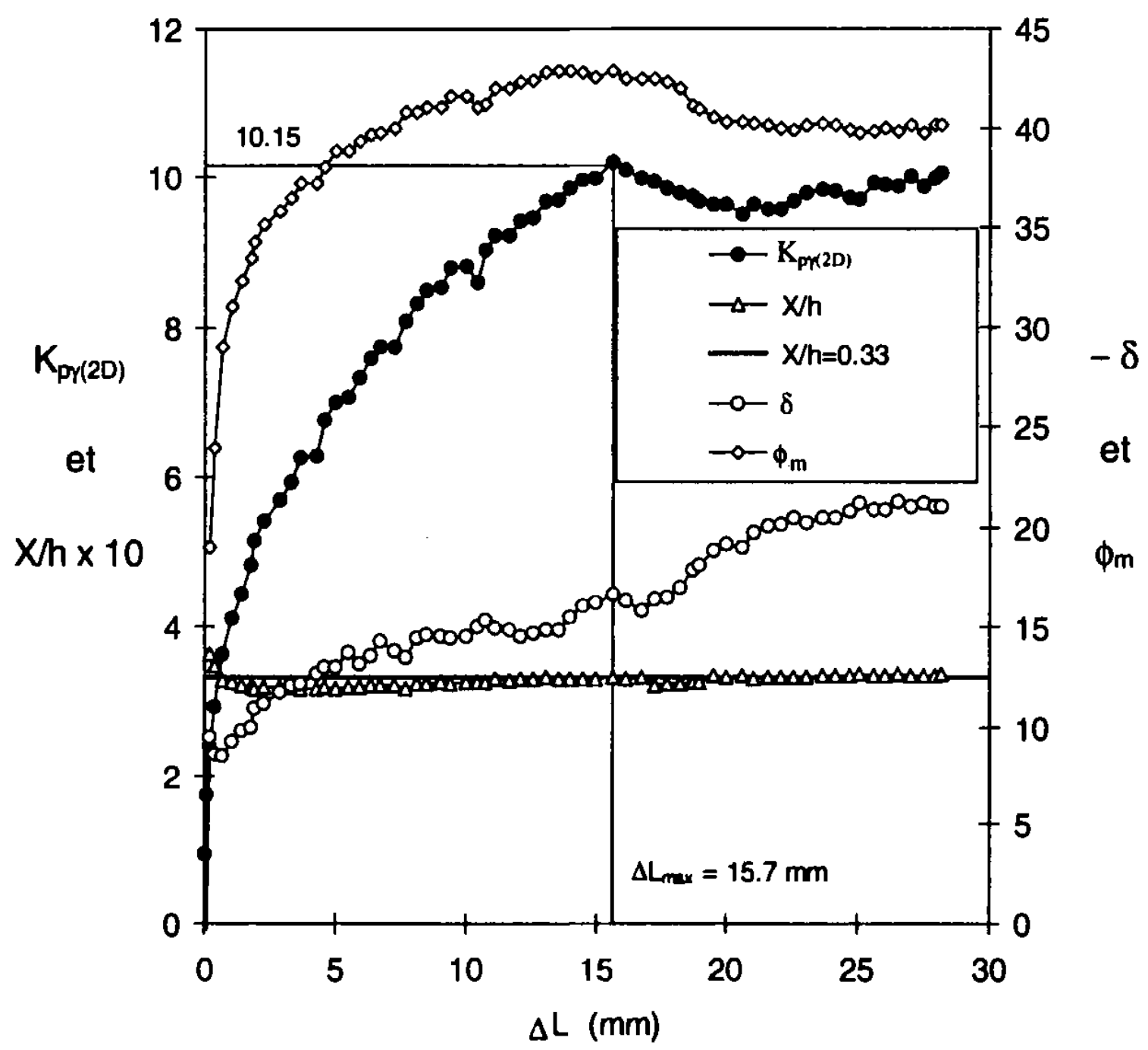

Figure 3. Evolution de $K_{p \times(2 D)}, X / h, \phi_{m}$ et $\delta$ en fonction de la translation $\Delta L$ de

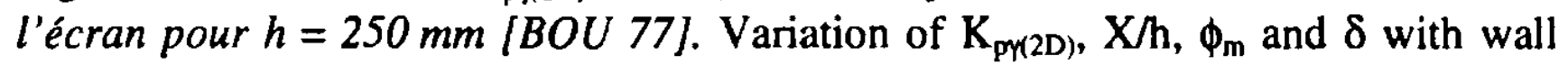
movement $\Delta \mathrm{L}$ for $\mathrm{h}=250 \mathrm{~mm}$ [BOU 77] 
Afin d'évaluer l'effet d'échelle du modèle utilisé pour cette étude, on analyse ici un essai bidimensionnel conduit par Bourdeau [BOU 77] sur un écran de $250 \mathrm{~mm}$ de fiche, suivant la procédure initiée par Rowe et Peaker [ROW 65] et reprise par Davis et Auger [DAV 79] et Héritier et Plumelle [HER 85].

La figure 3 présente l'évolution des différents paramètres de cet essai avec le déplacement de l'écran. Il s'agit d'une part du coefficient $K_{p \times 2 D)}$ déduit de l'effort résultant et du rapport adimensionnel $X / h$, qui sont représentés sur l'axe de gauche des ordonnées, d'autre part de $\delta$ et de l'angle de frottement mobilisé théorique $\phi_{m}$ qui sont représentés sur l'axe de droite des ordonnées. L'angle mobilisé $\phi_{m}$ est déduit des tables de Kérisel et Absi [KER 90] à partir des valeurs mesurées de $K_{p \times(2 D)}$ et de $\delta$.

Dans un grand modèle, la rupture progressive du sol le long de la surface de glissement explique que la résistance résiduelle puisse être atteinte au pied de l'écran alors que la résistance de pic n'est pas encore mobilisée en surface. Dans ces conditions, l'angle de frottement interne mobilisé $\phi_{m}$ reste inférieur à l'angle de frottement $\phi$ mesuré en laboratoire et peut se rapprocher de l'angle résiduel $\phi_{r}$. A l'inverse pour un petit modèle, l'effet conjugué d'une rupture progressive moins marquée et d'une dilatance relativement plus importante conduit à des valeurs de $\phi_{m}$ supérieures à $\phi$. Dans l'exemple de la figure $3, \phi_{m}$ atteint $43^{\circ}$ au pic de l'effort et se stabilise à $\phi_{m}=40^{\circ}$ pour de grands déplacements, alors que des essais de cisaillement direct conduisent à $\phi=34,5^{\circ}$ et $\phi_{r}=26,5^{\circ}$ pour $\gamma_{d}=15,6 \mathrm{kN} / \mathrm{m}^{3}$.

Selon l'interprétation de Kérisel [KER 72] et de Davis et Auger [DAV 79], l'influence de la taille du modèle et de la dilatance peut être exprimée par le coefficient $\frac{\phi_{m}-\phi_{r}}{\phi-\phi_{r}}$ qui est d'autant plus élevé que le modèle est petit. La valeur $\frac{\phi_{m}-\phi_{r}}{\phi-\phi_{r}}=2,1$ obtenue pour $h=250 \mathrm{~mm}$ est en accord avec l'analyse de ces auteurs.

Enfin, l'évolution croissante de $\delta$ avec le déplacement de l'écran, constatée sur de nombreux modèles, est un phénomène qui semble indépendant de la taille du modèle. Mais il apparaît acquis que $|\delta|$ ne peut cependant dépasser la valeur $\phi_{r}$ [KER 72].

En conclusion, l'analyse de cet essai montre un effet d'échelle non négligeable quant à l'intensité de la butée sur notre modèle. Ainsi, le coefficient de butée atteint la valeur $K_{p \times(2 D)}=10,15$ alors que sa valeur théorique, pour $\phi=34,5^{\circ}$ et $\delta=-16,7^{\circ}$ est $K_{p \gamma}=6,2$. Le facteur d'échelle pour cet écran, défini par $A=K_{p \times 2 D} / K_{p \gamma}$ est égal à 1,64. Dans ces conditions, il conviendra d'analyser nos résultats d'essais de butée tridimensionnelle, non pas en valeur absolue, mais en référence à la butée bidimensionnelle obtenue dans les mêmes conditions expérimentales. 


\subsection{Les mécanismes de mobilisation de la butée tridimensionnelle}

Pour deux écrans de section rectangulaire ayant une hauteur $h=200 \mathrm{~mm}$ et une épaisseur $a=30 \mathrm{~mm}$ identiques mais de largeurs différentes $b=100 \mathrm{~mm}$ et $b=15 \mathrm{~mm}$, les figures 4 et 5 montrent l'évolution des paramètres de la butée tridimensionnelle avec le déplacement de translation $\Delta L$. L'examen de ces figures prouve que le comportement de l'écran est très différent, tant qualitativement que quantitativement, selon qu'il est de type élancé ou peu élancé. La synthèse des résultats de Belabdelouhab [BEL 88] et Meksaouine [MEK 93] montre que la frontière entre les deux domaines correspond à une valeur critique $(b / h)_{c r}$ voisine de 0,25 .

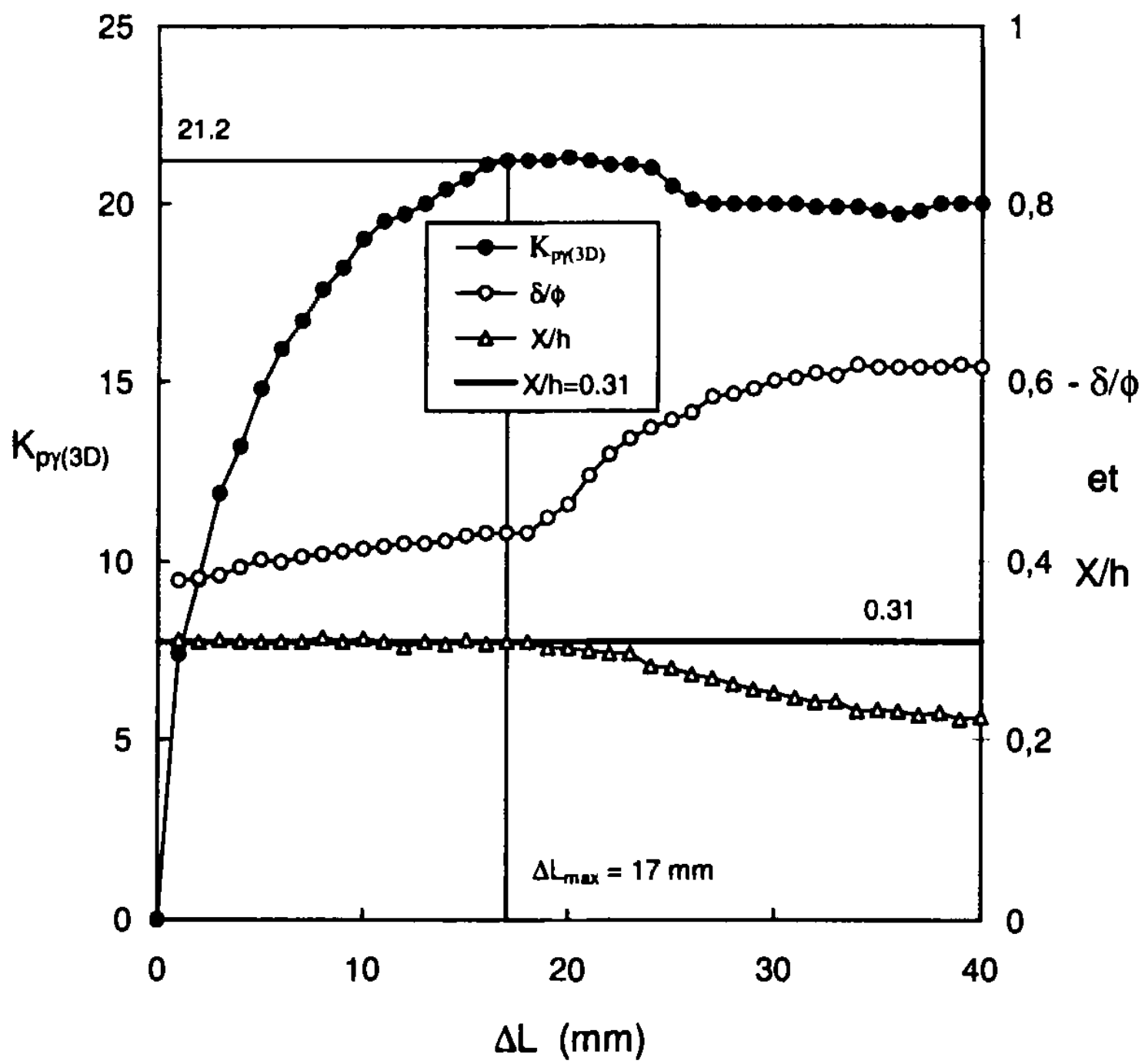

Figure 4. Evolution de $K_{p \times 3 D h} X / h$ et $\delta / \phi$ en fonction de la translation $\Delta L$ de l'écran pour $b / h=0,5$ avec $b=100$ et $h=200 \mathrm{~mm}$ [MEK 93]. Variation of $\mathrm{K}_{\mathrm{px}(3 \mathrm{D}),} \mathrm{X} / \mathrm{h}$ and $\delta / \phi$ with wall movement $\Delta \mathrm{L}$ for $\mathrm{b} / \mathrm{h}=0.5$ with $\mathrm{b}=100$ and $\mathrm{h}=200 \mathrm{~mm}$ [MEK 93]

Pour $b / h=0,5$ (figure 4), l'évolution des paramètres de la butée tridimensionnelle est proche du comportement observé pour un écran continu. La relation effortdéplacement est de type pic-palier et la croissance régulière de l'obliquité $\delta$ conduit à $|\delta / \phi|=0,43$ au pic, puis à $|\delta / \phi|=0,61$ au palier. Jusqu'au pic, qui est obtenu pour 
un déplacement relatif $\Delta L_{\text {ma }} h=8,5 \%$, l'effort résultant se stabilise légèrement sous le tiers inférieur de l'écran à la valeur $X / h=0,31$.

Pour $b / h=0,075$ (figure 5), en dehors de la bonne stabilité du point d'application à $X / h=0,3$, les autres paramètres de la butée tridimensionnelle sont en désaccord avec les observations précédentes. La relation $K_{\left.p\}^{3 D}\right)}-\Delta L$ est en effet de type palier et l'obliquité $\delta$ décroît régulièrement jusqu'au palier qui est atteint pour un déplacement relatif $\Delta L_{\operatorname{ma}} / h$ de $10 \%$. L'obliquité relative $|\delta / \phi|$ à la rupture est faible, de l'ordre de $18 \%$.

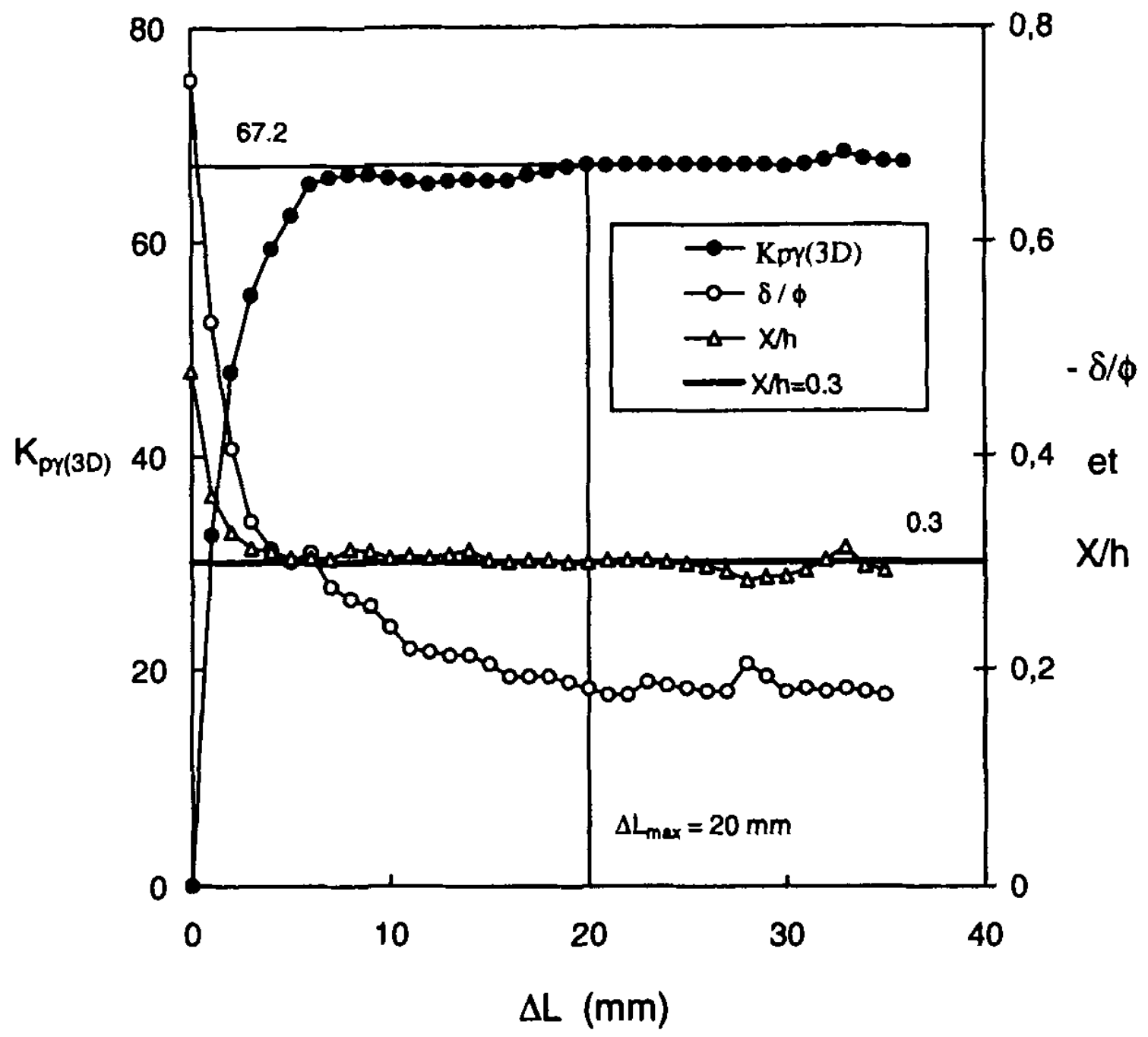

Figure 5. Evolution de $K_{p \nmid(3 D)}, X / h$ et $\delta / \phi$ en fonction de la translation $\Delta L$ de l'écran pour $b / h=0,075$ avec $b=15$ et $h=200 \mathrm{~mm}$ [MEK 93]. Variation of $\mathrm{K}_{\mathrm{py}(3 \mathrm{D}),} \mathrm{X} / \mathrm{h}$ and $\delta / \phi$ with wall movement $\Delta \mathrm{L}$ for $\mathrm{b} / \mathrm{h}=0.075$ with $\mathrm{b}=15$ and $\mathrm{h}=200 \mathrm{~mm}$ [MEK 93]

Les observations sur modèles montrent que ces différences de comportement sont liées à un changement de mécanisme :

- pour un écran peu élancé $(h / b<4)$, la translation de l'écran provoque un mécanisme de rupture semblable au mécanisme bidimensionnel avec un prisme de rupture tridimensionnel partant de la base de l'écran, tel que représenté sur la figure $6 \mathrm{a}$; 
- pour un écran d'élancement important $(h / b>4)$, le prisme de rupture tridimensionnel ne concerne que la partie supérieure de l'écran (figure 6b). Dans sa partie inférieure, la translation de l'écran provoque un refoulement latéral du sol, ce qui explique les faibles valeurs de $\delta / \phi$ constatées dans ces conditions.

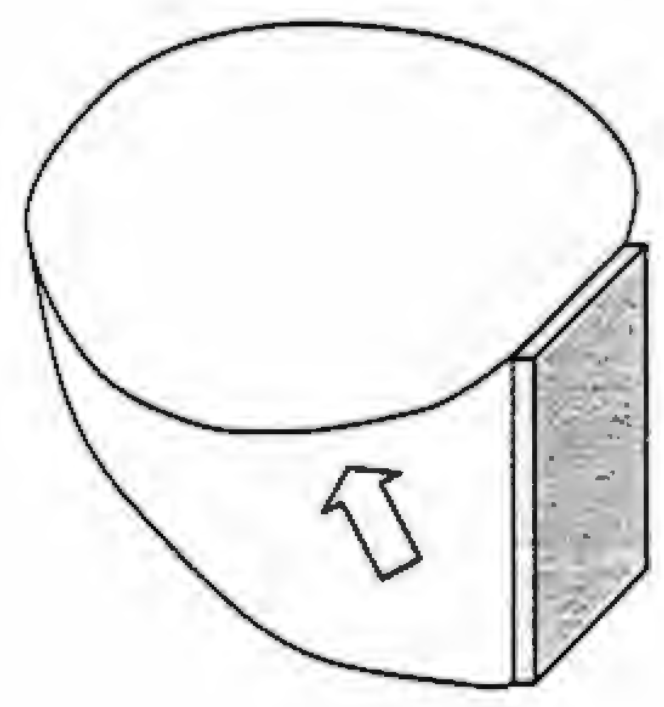

a

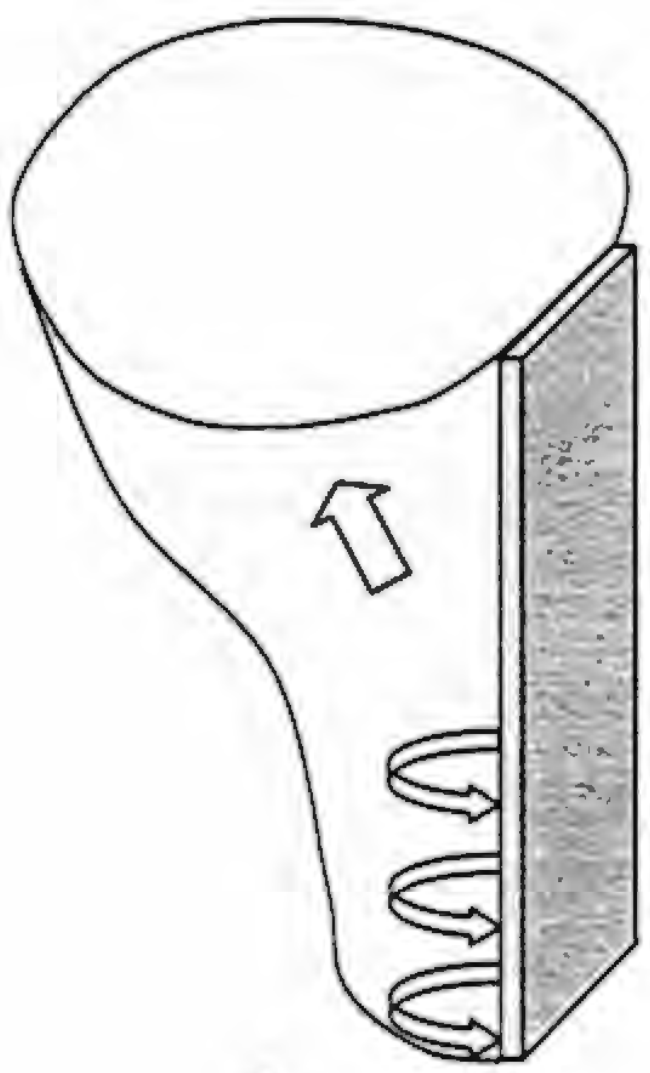

b

Figure 6. Mécanismes de rupture observés : a) cas d'un écran peu élancé ; b) cas d'un écran élancé. Observed failure mechanisms : a) case of a large wall ; b) case of a slender wall

2.4. Influence de l'élancement de l'écran sur le coefficient de butée tridimensionnelle

Les travaux antérieurs sur la butée tridimensionnelle indiquent que l'effort total de butée peut être décomposé en une action à caractère bidimensionnel sur la face avant de l'écran et une réaction du massif au contact des faces latérales du prisme de rupture. Selon Horn [HOR 72] la relation [1] entre le coefficient de butée tridimensionnelle $K_{p Y 3 D}$, le coefficient de butée bidimensionnelle $K_{p \gamma}$ et l'élancement de l'écran $h / b$ est à la fois dépendant du facteur de modèle $A$ et d'un facteur de forme $C$, comme suit :

$$
K_{p \gamma(3 D)}=K_{p \gamma} A\left(1+C \frac{h}{b}\right)
$$


Zweck [ZWE 53], Weissenbach [WEI 61] et Horn [HOR 67, 72] ont particulièrement étudié le problème de la butée sur un écran de largeur limitée. L'analyse effectuée par Horn [HOR 72] de ces expériences et de celles réalisées avec des plaques enterrées par Buchholz [BUC 30], Petermann [PET 33], Hüeckel [HUE 57] et Brinch Hansen [BRI 66] montre une très forte dispersion entre résultats (figure 7).

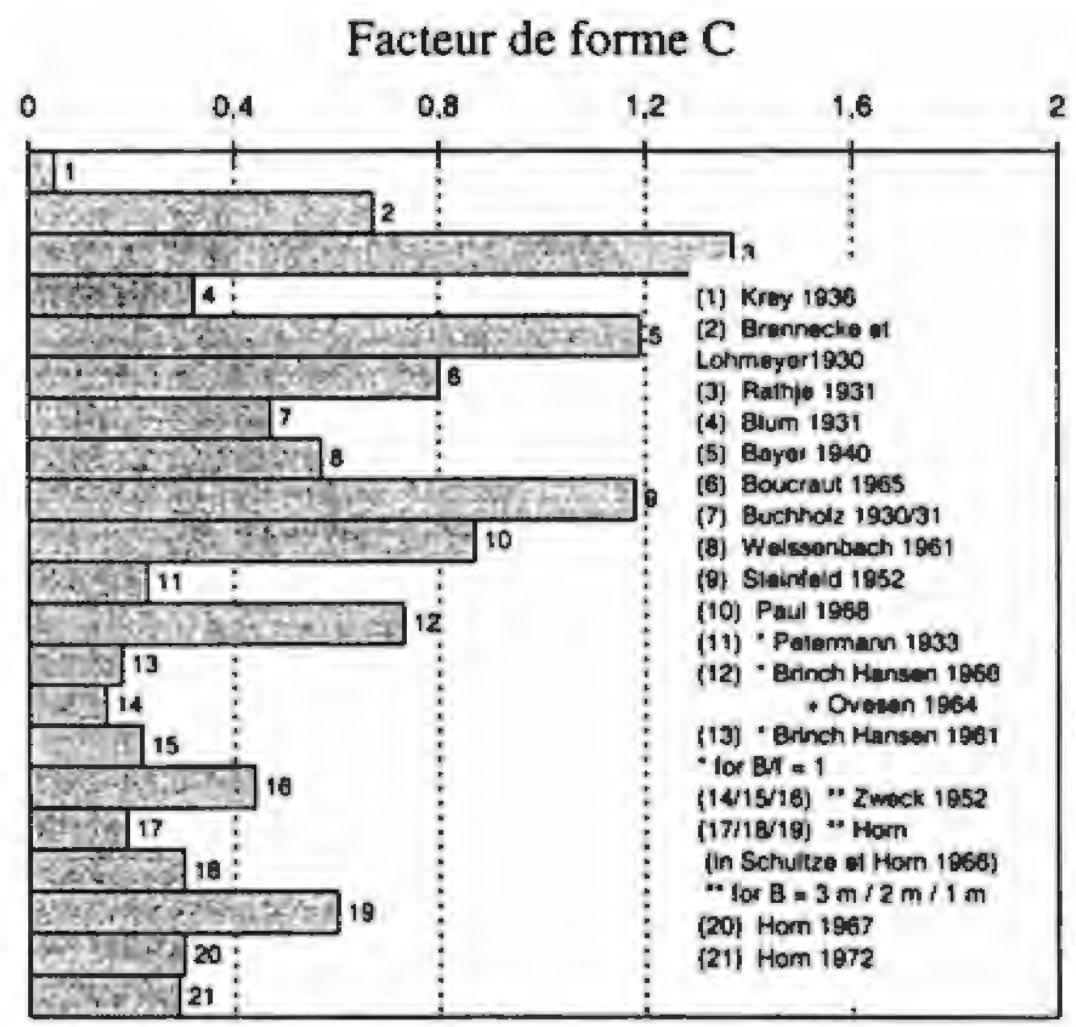

Figure 7. Valeurs du facteur de forme C [HOR 72]. Values of shape factor C [HOR 72]

L'association de modèles réduits bidimensionnel et tridimensionnel de taille identique permet de s'affranchir du facteur de modele dans l'interprétation des résultats. Ainsi, la relation [1] peut être remplacée par la formule simplifiée [2] :

$$
K_{p r(30)}=K_{p r(2 D)}\left(1+C \frac{h}{b}\right)
$$

avec $K_{P Y 2 D)}=10,15$ déterminé sur un écran continu de même hauteur.

La corrélation entre $K_{p(3 D)}$ et le rapport $b / h$ (figure 8) est établie pour tous les types d'écrans expérimentés par Belabdelouhab [BEL 88] et Meksaouine [MEK 93]. Elle est completée par quelques résultats obtenus par Weissenbach [WEI 61] pour des conditions expérimentales voisines, tant pour la géométrie des écrans que pour la granulométrie et la densité relative de mise en place d'un sable sec. Ces résultats montrent un changement de comportement correspondant a la valeur critique $(b / h)_{c r}=0,25$. Pour $b / h<0,25$ le fuseau des points de mesure vérifiant la relation [2] 
correspond à $0,3<C<0,4$ alors que ce facteur de forme est compris entre 0,4 et 0,8 pour $b / h>0,25$.

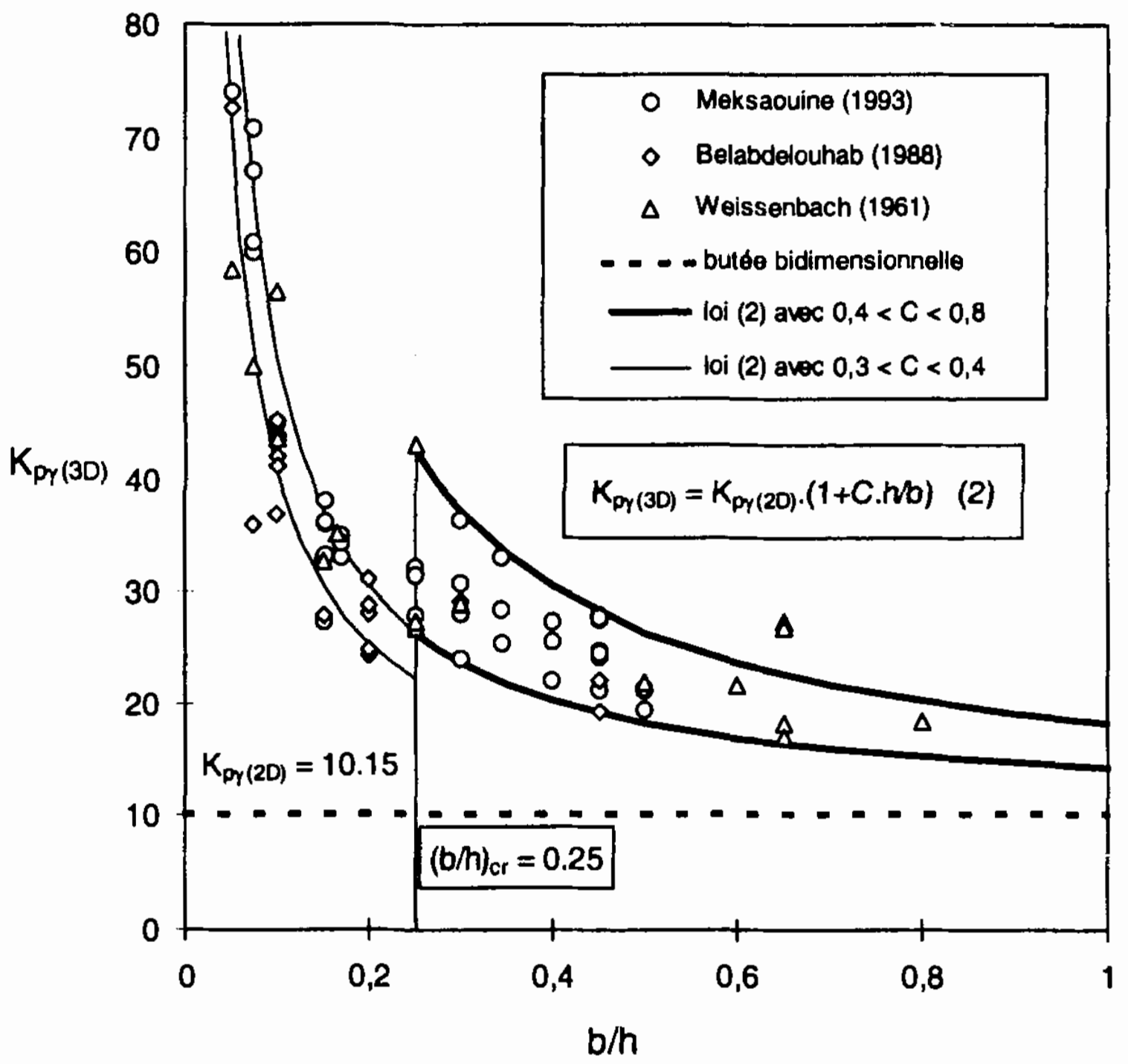

Figure 8. Relation entre le coefficient de butée $K_{p \times(3 D)}$ et le rapport b/h de l'écran pour $10<b(\mathrm{~mm})<130$ et $50<h(\mathrm{~mm})<400$. Relationship between $\mathrm{K}_{\mathrm{py}(3 \mathrm{D})}$ and $\mathrm{b} / \mathrm{h}$ for $10<b(\mathrm{~mm})<130$ and $50<\mathrm{h}(\mathrm{mm})<400$

La dispersion constatée peut être expliquée par des différences de conditions expérimentales: légers écarts sur la densité relative de mise en place du sol, différences de taille des modèles (hauteur de l'écran comprise entre 125 et $400 \mathrm{~mm}$ ).

Si l'on s'intéresse aux seules valeurs situées en deçà de l'élancement critique $(b / h>0,25)$ pour lesquelles nous proposons un schéma de calcul, le facteur $C$ moyen serait de l'ordre de 0,6 .

\subsection{Etude de la surface débouchante}

La corrélation entre les paramètres géométriques $b$ et $h$ de l'écran d'une pars s, les développements $L$ et $V$ de la surface débouchante (figure 1) d'autre part, permet de 
compléter l'analyse de l'effet de l'élancement de l'écran sur les dimensions de la surface débouchante. La figure 9 montre que la forte croissance de $L / h$ avec $b / h$ est limitée au domaine des écrans élancés $(b / h<0,25)$. Cette progression s'atténue dans le domaine des écrans larges $(b / h>0,25)$ et ne semble pas devoir dépasser la valeur asymptotique $L / h=2,5$ pour $b / h>1,5$.

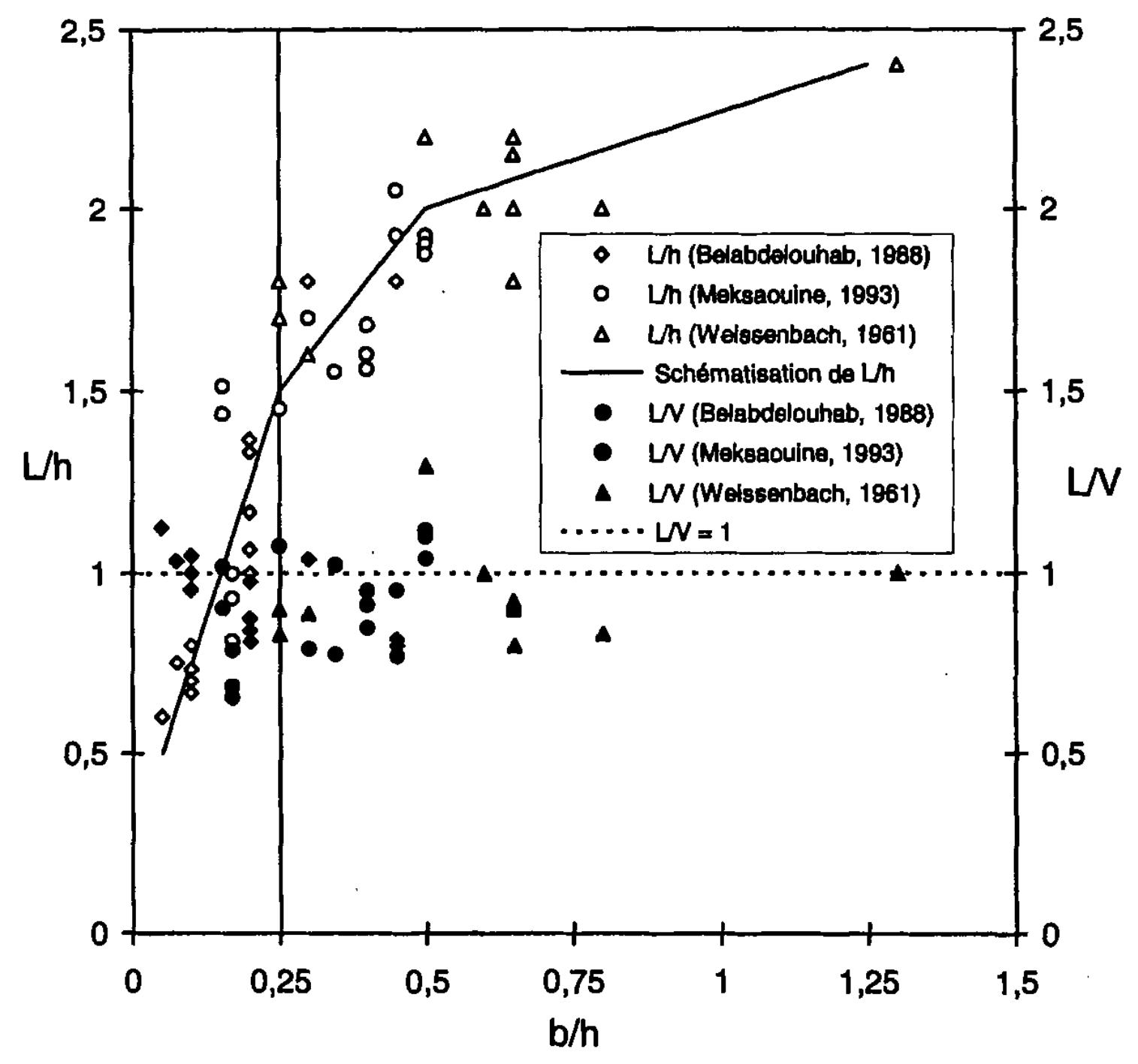

Figure 9. Dimensions en plan de la surface débouchante pour $10<b(\mathrm{~mm})<130$ et $50<h(\mathrm{~mm})<400$. Dimensions of the failure mechanism at the ground surface for $10<\mathrm{b}(\mathrm{mm})<130$ and $50<\mathrm{h}(\mathrm{mm})<400$

Par ailleurs, malgré une certaine dispersion des points de mesure ne permettant pas de préciser une loi d'évolution du rapport $L N$, il s'avère néanmoins que le développement frontal $L$ est globalement inférieur au développement latéral $V$.

\subsection{Conclusions de l'étude expérimentale}

Cette étude a permis de mettre en évidence deux modes de rupture distincts suivant l'élancement de l'écran. De plus, les résultats expérimentaux ont permis de préciser une formulation empirique de la butée tridimensionnelle fonction d'un facteur de 
forme $C$, de l'élancement de l'écran $h / b$ et des résultats classiques de la butée bidimensionnelle. Dans la suite de cet article, on se propose de présenter un modèle de calcul de la butée tridimensionnelle dans le cas des écrans d'élancement faible $(h / b<4)$. Le modèle présenté est basé sur la méthode cinématique de la théorie de l'analyse limite.

\section{Calcul de la butée sur un écran de forme rectangulaire par l'approche cinématique de l'analyse limite}

L'étude des problèmes de stabilité par la théorie de l'analyse limite (Méthodes statique et cinématique) a fait l'objet de nombreux travaux : [ABD 94, 97], [CHE 75], [DEB 93, 98], [PAS 78, 82], [REG 99], [SAL 90, 93], [SOU 97, 98a, 98b, 99a, 99b]. Ces méthodes permettent de déterminer la charge de rupture par deux approches basées sur l'application des théorèmes limites de Drucker et Prager [DRU 52], dont le principe de base a été présenté dans Salençon [SAL 83].

L'étude de la butée bidimensionnelle par une approche statique a fait l'objet d'un nombre d'études limité. On peut citer les travaux de Lysmer [LYS 70], Lee et Herington [LEE 72] et ceux de Basudhar et Singh [BAS 79]. En revanche, l'approche cinématique a été utilisée par plusieurs auteurs comme Finn [FIN 67], Chen et Scawthorn [CHE 70] et Chen et Rosenfarb [CHE 73]. Ces derniers ont présenté les travaux les plus significatifs dans ce domaine en étudiant six mécanismes de rupture translationnels allant du mécanisme le plus simple de Coulomb, aux mécanismes plus évolués (mécanisme à deux blocs rigides et mécanisme en log-sandwich). D'autre part, Soubra et al. [SOU 99b] ont proposé un schéma de calcul de la butée bidimensionnelle basé sur un mécanisme rotationnel délimité par une spirale logarithmique d'angle $\phi$. Dans le cadre des mécanismes translationnels, le mécanisme en log-sandwich donne souvent le meilleur majorant de l'effort de butée. Le mécanisme à deux blocs rigides donne des résultats très proches de ceux donnés par le mécanisme en log-sandwich. Ces mécanismes fournissent à l'heure actuelle la borne supérieure la plus faible disponible dans la littérature pour les mécanismes bidimensionnels de type translationnel. L'objectif de cet article est la généralisation des mécanismes translationnels à un ou deux blocs rigides envisagés par Chen et Rosenfarb [CHE 73] au cas de la butée tridimensionnelle. Trois mécanismes de rupture sont proposés. La confrontation des résultats entre mécanismes permet de fournir le meilleur majorant de l'effort de butée tridimensionnelle.

\subsection{Présentation du problème}

Dans l'étude présente, nous considérons le cas d'un écran vertical de forme rectangulaire (figure 10). Cet écran est défini par sa hauteur $h$ et sa largeur $b$. Il met 
en butée un massif de sol frottant homogène et isotrope limité par une surface libre horizontale.

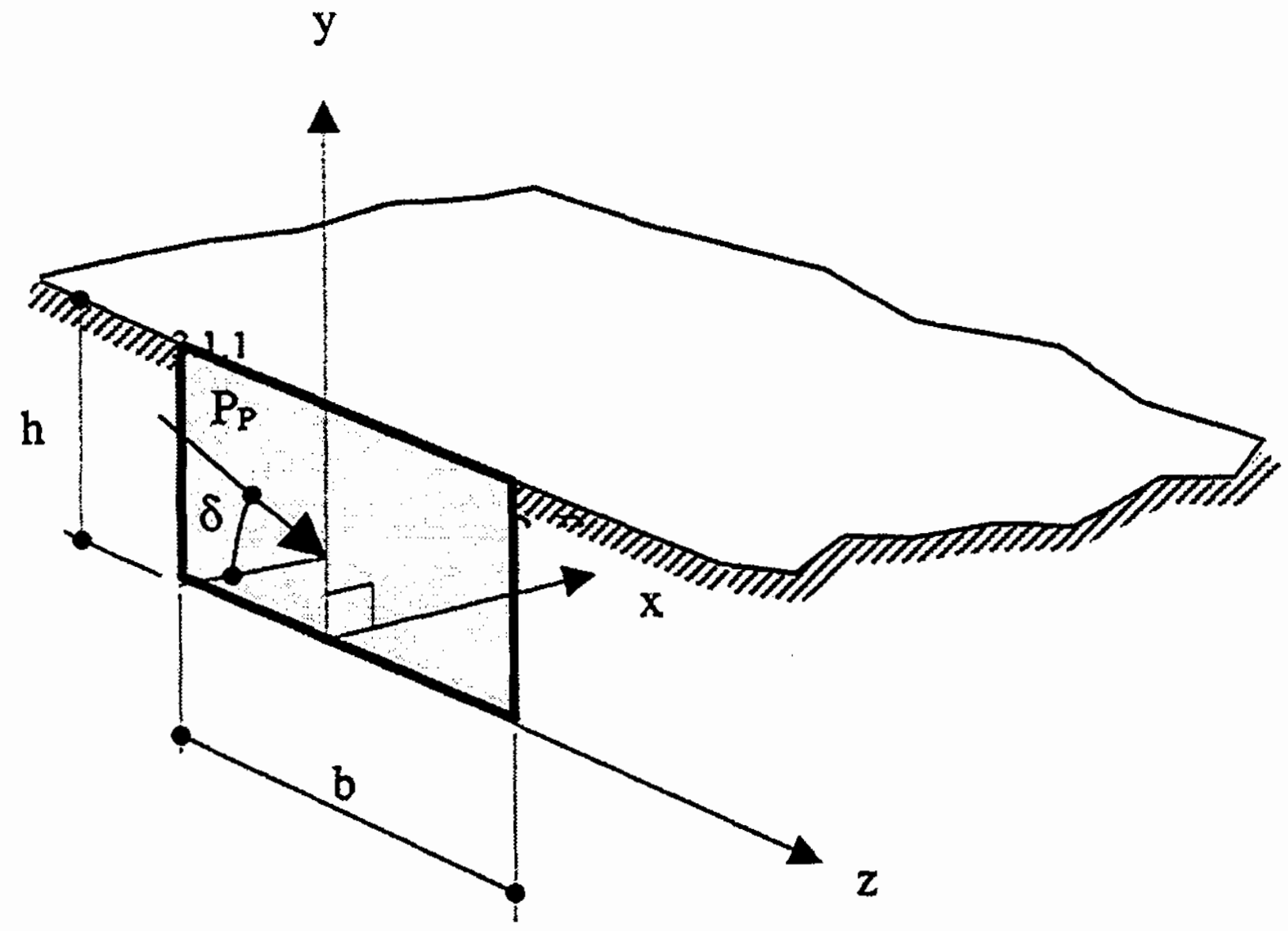

Figure 10. Présentation du problème et notations. Presentation of the problem and notations

\subsection{Mécanismes de rupture}

Dans ce paragraphe, nous présentons les trois mécanismes de rupture envisagés. Pour ne pas alourdir la présentation, nous donnons uniquement une description succincte de chacun de ces trois mécanismes de rupture. Les détails des calculs nécessaires à leurs constructions figurent dans Regenass [REG 99].

\subsubsection{Mécanisme à un bloc rigide $M 1$}

Le mécanisme $M I$ est le mécanisme le plus simple permettant la prise en compte de l'effet tridimensionnel. Ce mécanisme s'inspire du schéma de rupture proposé par Blum [BLU 32] tout en respectant les conditions cinématiques de l'analyse limite. Il constitue également une adaptation du mécanisme bidimensionnel à un bloc rigide proposé par Chen et Rosenfarb [CHE 73] dans le cadre de l'approche cinématique de l'analyse limite. 


\subsubsection{Description géométrique}

Le mécanisme $M I$ est composé d'un bloc rigide symétrique $A A^{\prime} B B^{\prime} D D^{\prime}$ de forme pentaédrique contigu à l'écran $A A^{\prime} B B^{\prime}$ ' (Agure 11a). Ce bloc est délimité à sa base
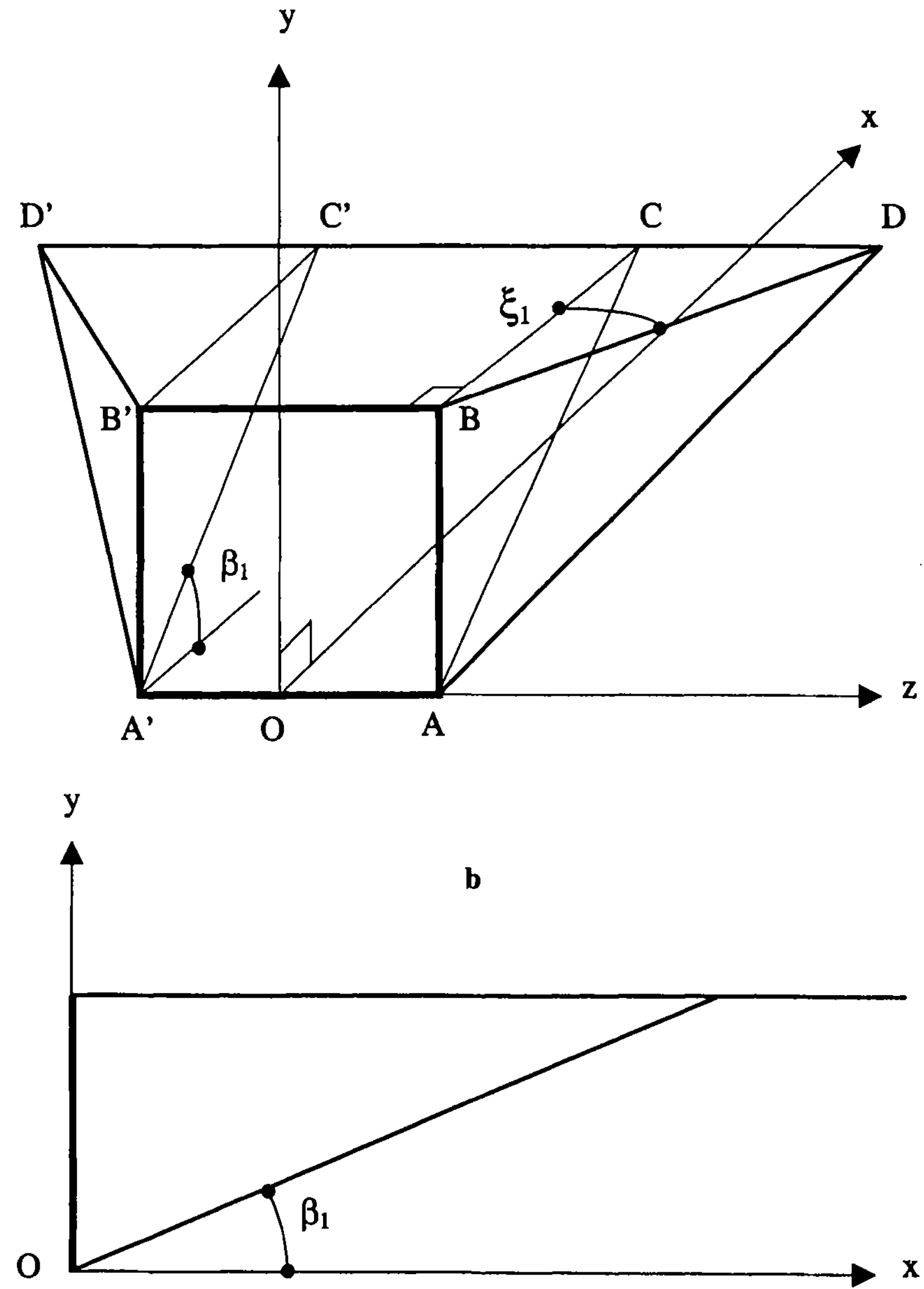

Figure 11. a) Mécanisme de rupture $M I$; b) Coupe du mécanisme suivant Oxy. a) Failure mechanism $\mathrm{M1}$; b) Cross-section through Oxy 
par le plan $A A^{\prime} D D^{\prime}$ et latéralement par les faces triangulaires $A B D$ et $A^{\prime} B^{\prime} D^{\prime}$ passant par les arêtes verticales de l'écran $A B$ et $A^{\prime} B^{\prime}$. Il débouche à la surface du sol suivant le trapèze $B B^{\prime} D D^{\prime}$. Nous présentons (figure $11 \mathrm{~b}$ ) la coupe du mécanisme de rupture suivant le plan vertical de symétrie $O x y$. Ce mécanisme est défini par le seul paramètre angulaire $\beta_{l}$, l'angle dièdre entre le plan horizontal et le plan de base $A A^{\prime} D D^{\prime}$.

L'orientation des plans de rupture du mécanisme $M 1$ n'est pas arbitraire. L'approche cinématique en analyse limite impose un angle égal à $\phi$ entre le vecteur vitesse du bloc et toutes les surfaces de discontinuité de vitesses. Par rapport au plan de base, cette condition impose la direction du vecteur vitesse $V_{l}$ du bloc, orientée à $\beta_{I}+\phi$ par rapport à l'horizontal. En ce qui concerne le plan latéral $A B D$, cette condition permet de fixer son ouverture par rapport au plan $A B C$ parallèle au plan $O x y$. L'angle dièdre $\xi_{l}$ (figure 1 la) entre le plan latéral $A B D$ et le plan $A B C$ est obtenu en imposant un angle égal à $\phi$ entre le vecteur vitesse $V_{l}$ du bloc et sa projection orthogonale sur le plan latéral $A B D$. L'expression de l'angle dièdre $\xi_{l}$ est donnée par :

$$
\operatorname{tg} \xi_{1}=\frac{\sin \phi}{\sqrt{\cos ^{2} \phi-\sin ^{2}\left(\beta_{1}+\phi\right)}}
$$

Le paramètre angulaire $\beta_{l}$ est soumis à deux conditions restrictives. D'une part, $\beta_{l}$ doit être supérieur à zéro pour permettre au bloc de sol en rupture de déboucher à la surface libre du sol. D'autre part, cet angle doit être inférieur à $\pi / 2-2 \phi$ pour rester dans le domaine de définition de la fonction donnée par l'expression [3]. La limite supérieure de l'angle $\beta_{1}$ correspond alors à l'épanouissement des plans latéraux dans le plan de l'écran.

\subsubsection{Etude de la cinématique du mécanisme $M I$}

Le mouvement horizontal de l'écran de vitesse $V_{0}$ induit un mouvement en translation du bloc rigide $A A^{\prime} B B^{\prime} D D^{\prime}$ de vitesse $V_{I}$ (figure 12). La vitesse de discontinuité $V_{0,1}$ à l'interface sol-écran est supposée tangente à l'écran conformément aux travaux de Chen [CHE 75]. D'autres auteurs ont adopté une vitesse inclinée de $\delta$ par rapport à l'écran afin de respecter la loi de normalité (cf. Mroz \& Drescher [MRO 69] et Collins [COL 69, 73]). Ces deux hypothèses aboutissent strictement à la même charge de rupture (cf. Drescher \& Detournay [DRE 93] et Michalowski [MIC 99]). On peut noter que toutes ces vitesses sont colinéaires au plan vertical de symétrie $O x y$. 

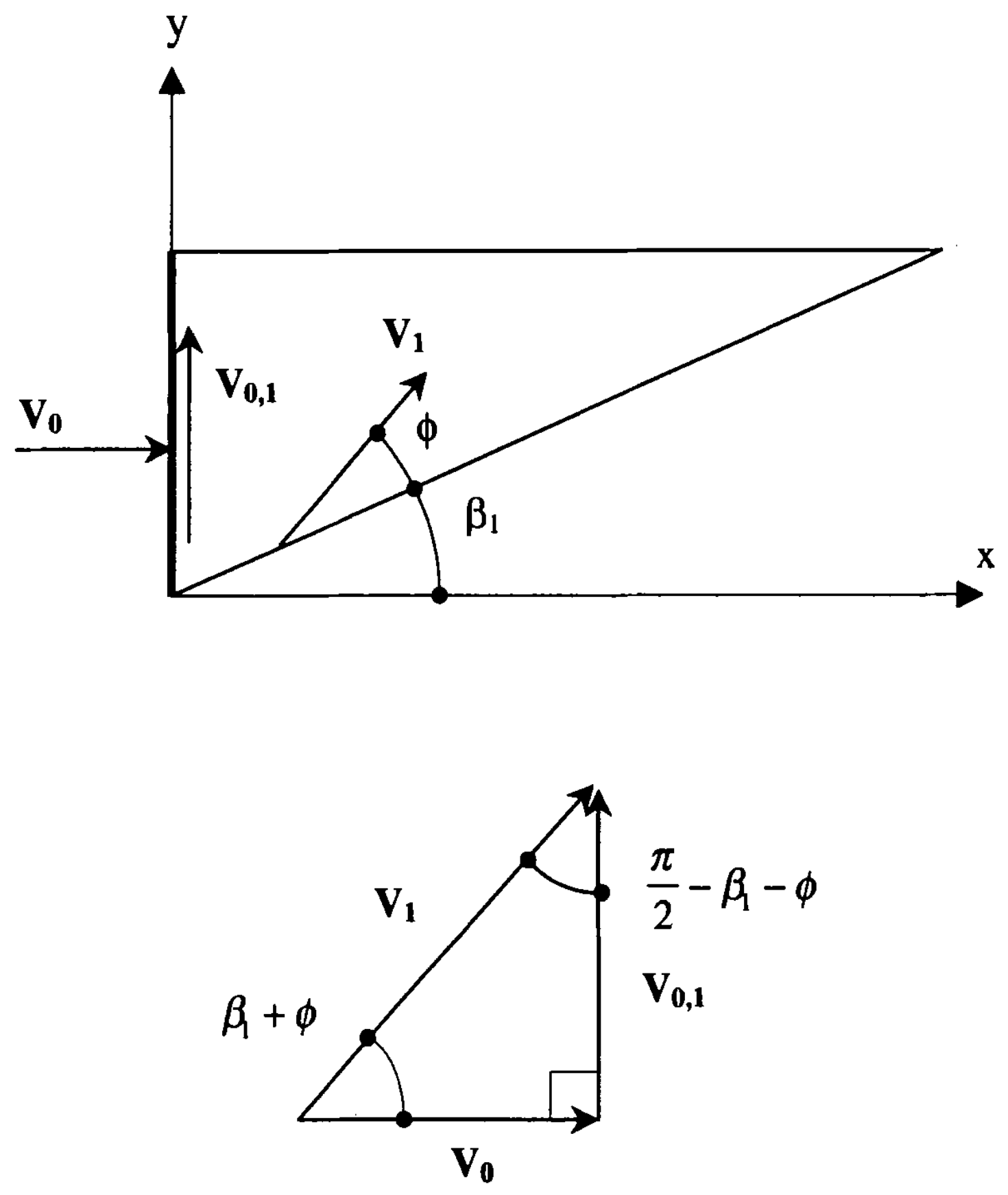

Figure 12. Cinématique du mécanisme MI et hodographe de vitesses. Kinematics of the Ml mechanism and velocity hodograph

\subsubsection{Mécanisme à deux blocs rigides $M 2$}

Le mécanisme à deux blocs rigides se révèle l'un des mécanismes les plus pertinents parmi les six mécanismes de rupture envisagés par Chen et Rosenfarb [CHE 73] dans le cas des écrans filants. Le mécanisme présent en constitue une adaptation tridimensionnelle.

\subsubsection{Description géométrique du mécanisme}

Le mécanisme $M 2$ est défini par deux blocs rigides pentaédriques symétriques par rapport au plan $O x y$, dont l'arête commune s'appuie sur le bord supérieur de l'écran $B B^{\prime}$ (figure 13). 
a

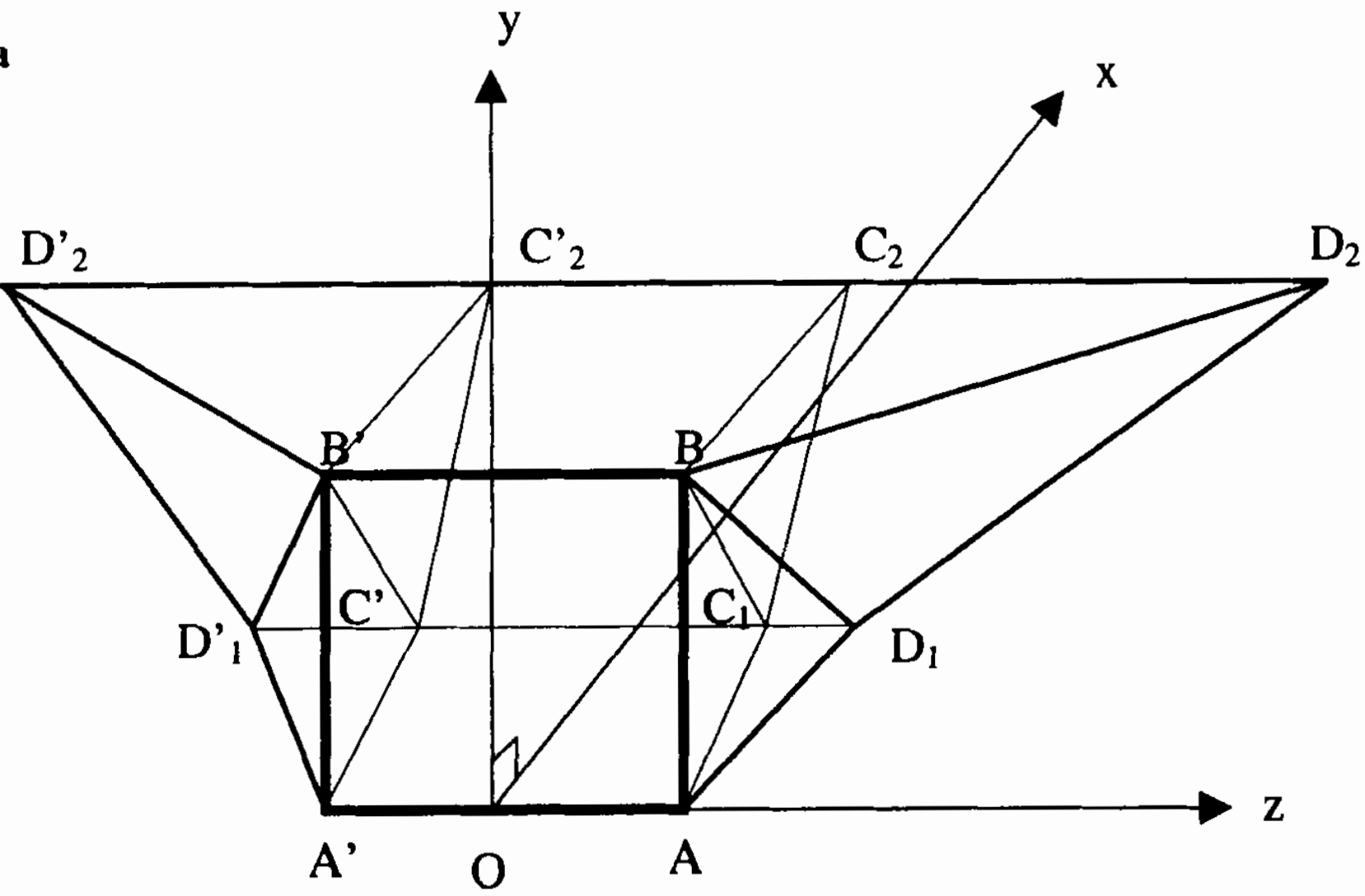

b

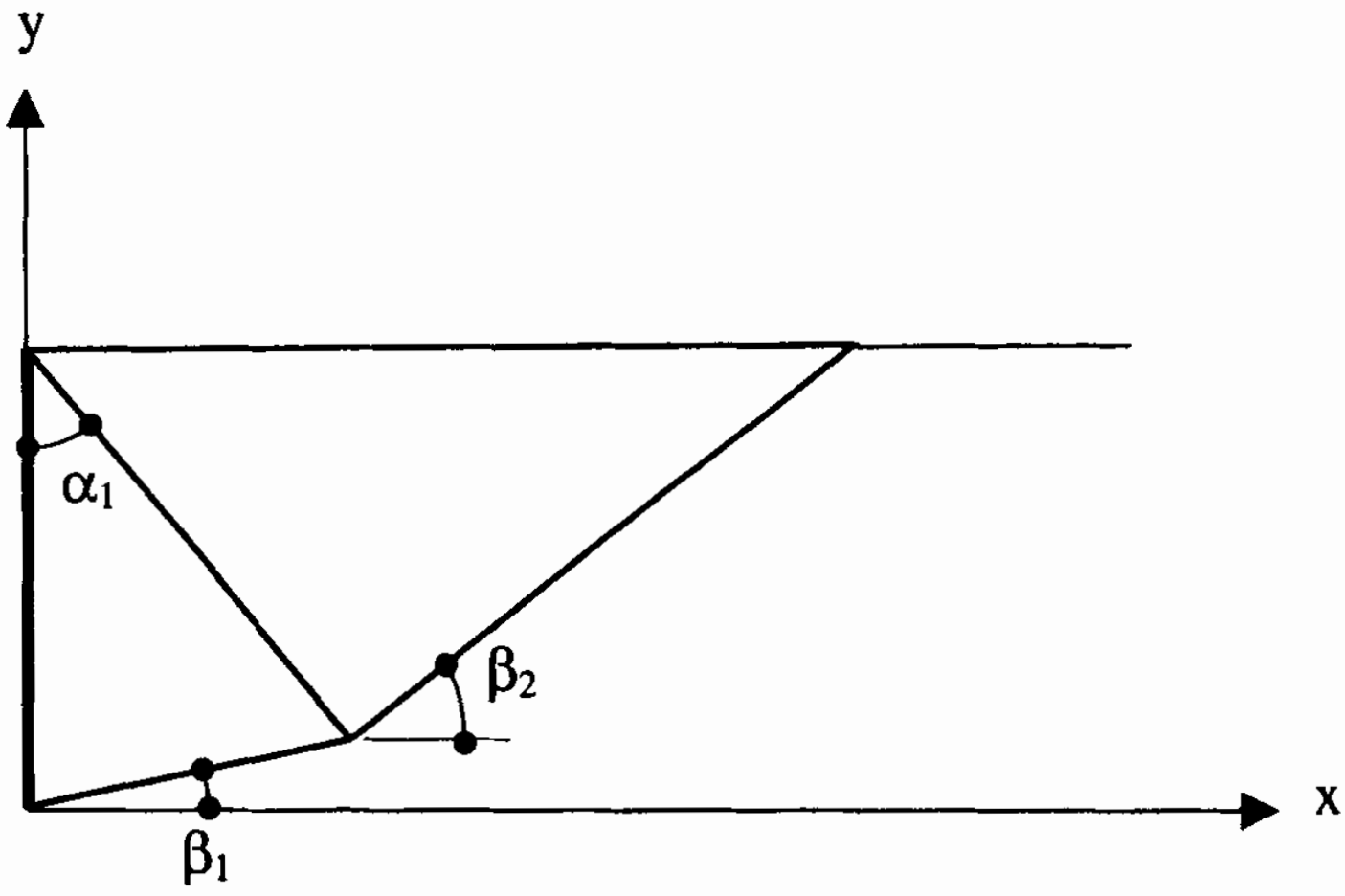

Figure 13. a) Mécanisme de rupture $M 2$; b) Coupe du mécanisme suivant Oxy a) Failure mechanism M2 ; b) Cross-section through Oxy

Le premier bloc $A A^{\prime} B B^{\prime} D_{1} D^{\prime}$ ', est contigu à l'écran $A A^{\prime} B B^{\prime}$ et est délimité par le plan de séparation $B B^{\prime} D_{1} D^{\prime}{ }_{1}$, le plan de base $A A^{\prime} D_{1} D^{\prime}{ }_{1}$ et les deux plans latéraux $A B D$, et $A^{\prime} B^{\prime} D^{\prime}$, (figure 13a). Le second bloc débouche à la surface du sol suivant 
le trapèze $B B^{\prime} D_{2} D_{2}{ }_{2}$. La coupe du mécanisme de rupture, effectuée suivant le plan vertical de symétrie $O x y$, est représentée sur la figure $13 \mathrm{~b}$.

Ce mécanisme est défini par trois paramètres angulaires :

$-\alpha_{l}$, l'angle entre l'écran et le plan de séparation $B B^{\prime} D_{l} D^{\prime}{ }_{l}$ des deux blocs ;

$-\beta_{i}(i=1,2)$, l'angle dièdre entre le plan de base du bloc $i$ et le plan horizontal.

Comme précédemment, chaque bloc doit respecter la condition cinématique qui impose l'orientation des faces délimitant ce bloc. Pour le premier bloc, les orientations des plans latéraux $A B D_{l}$ et $A^{\prime} B^{\prime} D^{\prime}{ }_{l}$ et du plan de base $A A^{\prime} D_{l} D^{\prime}{ }_{l}$, sont identiques à celles du mécanisme $M I$. En ce qui concerne le bloc supérieur, l'orientation des plans latéraux $B D_{1} D_{2}$ et $B{ }^{\prime} D^{\prime}{ }_{1} D^{\prime}{ }_{2}$ est obtenue, comme dans le cas du premier bloc, en imposant un angle égal à $\phi$ entre le vecteur vitesse $V_{2}$ et sa projection orthogonale sur les deux plans latéraux.
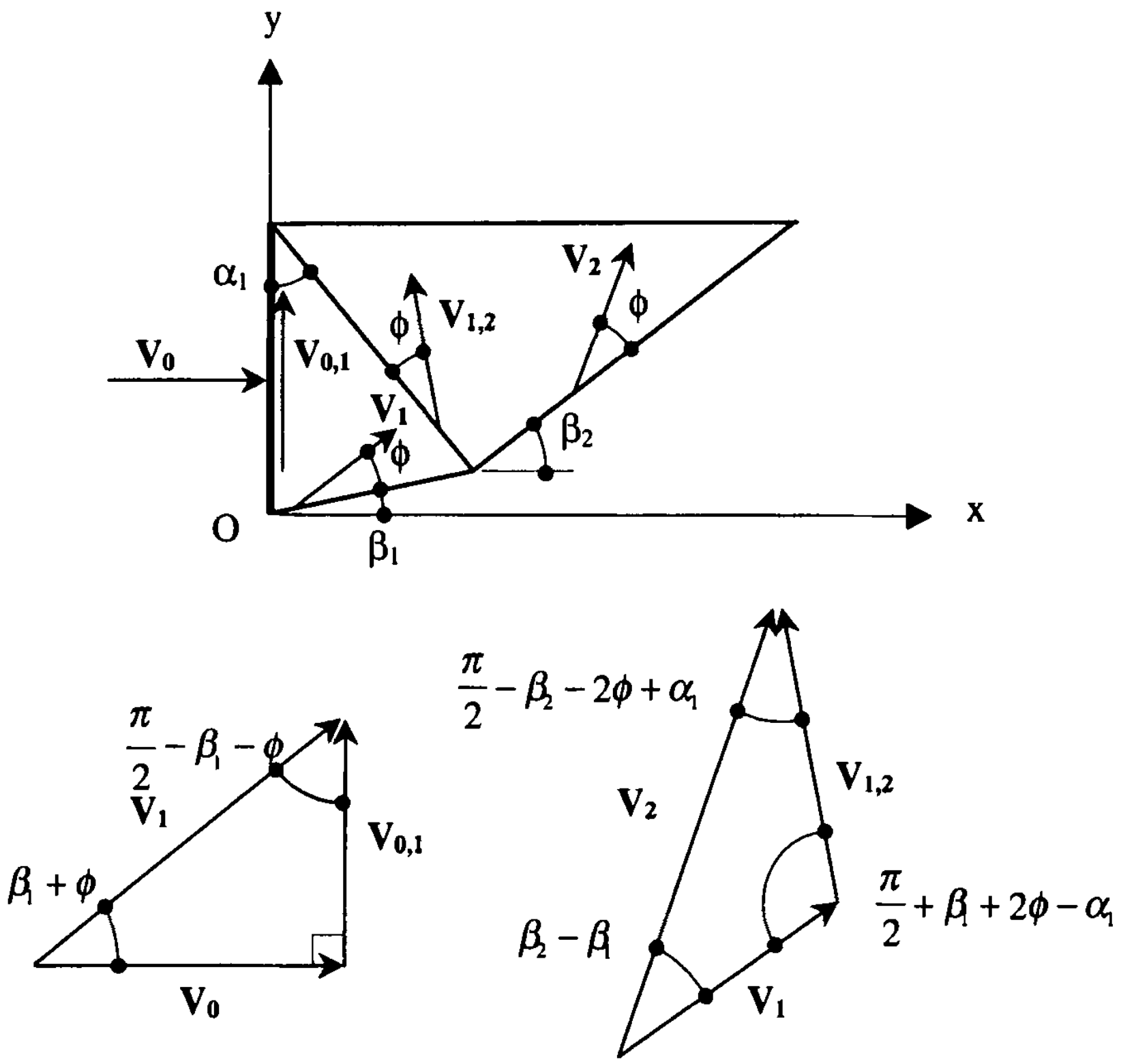

Figure 14. Cinématique du mécanisme $M 2$ et hodographes de vitesses. Kinematics of the M2 mechanism and velocity hodographs 
Contrairement au cas du monobloc, l'angle $\beta_{l}$ caractérisant l'orientation du plan de base du premier bloc n'est plus soumis à la condition $\beta_{l}>0$. En revanche, la limitation $\beta_{1}<\pi / 2-2 \phi$ subsiste.

\subsubsection{Etudẹ de la cinématique du mécanisme}

Comme dans le cas du mécanisme $M I$, les vitesses des blocs $V_{i}(i=1,2)$ et la vitesse de discontinuité entre blocs $V_{l, 2}$ appartiennent au plan vertical de symétrie $O x y$. Les hodographes de vitesses sont présentés sur la figure 14 .

\subsubsection{Mécanisme d̀ un bloc tronqué MIt}

Le mécanisme $M I t$ offre une amélioration du mécanisme $M I$ présenté précédemment. Il permet de réduire de manière significative le volume du sol mis en jeu lors de la rupture.

\subsubsection{Description du mécanisme}

Le mécanisme de rupture $M I t$ est composé d'un unique bloc rigide $A A^{\prime} B B^{\prime} M M^{\prime} C C^{\prime}$ (figure 15).

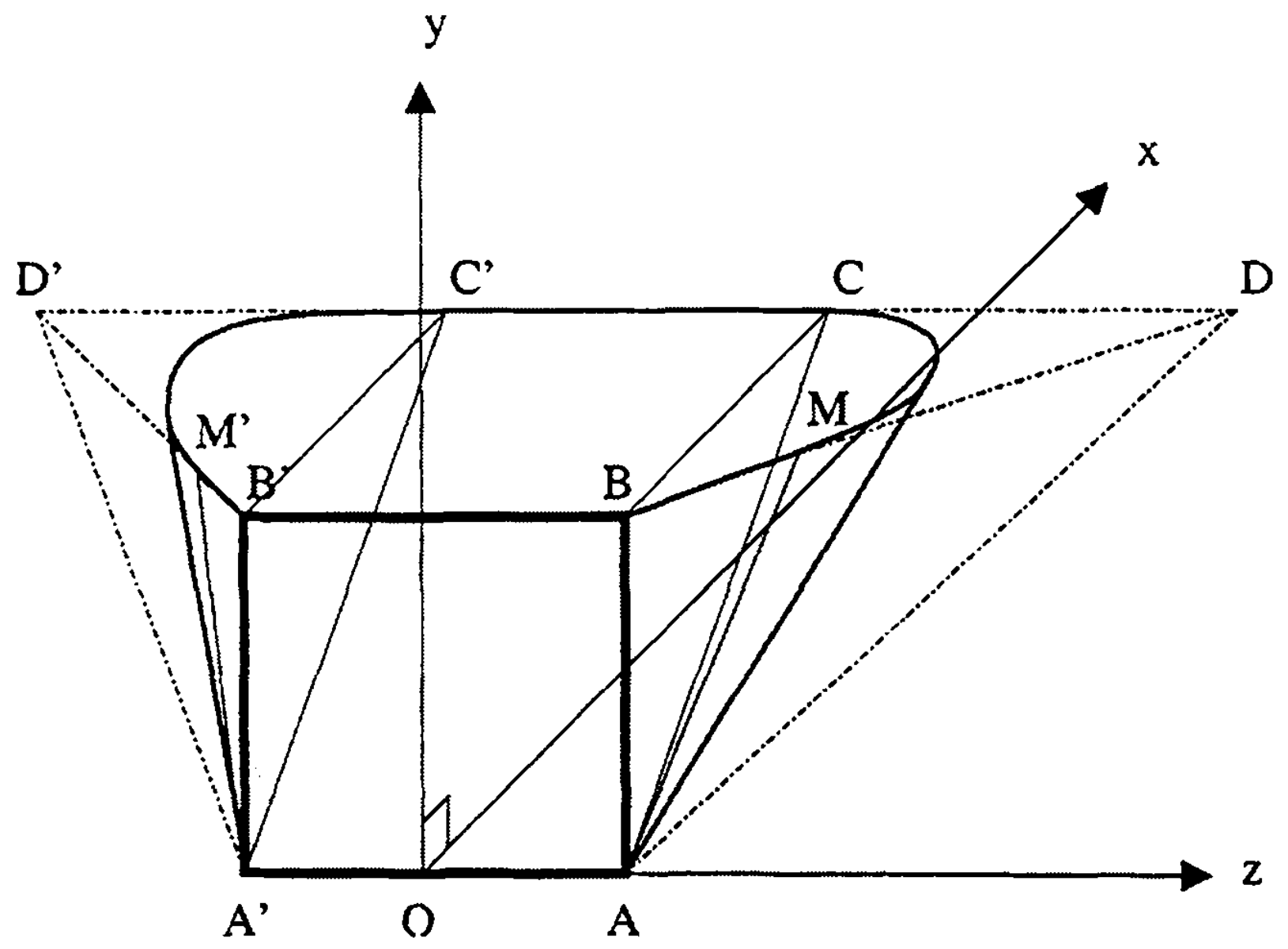

Figure 15. Mécanisme de rupture MIt. Failure mechanism MIt

Le plan de base $A A^{\prime} D D^{\prime}$ et les plans latéraux $A B D$ et $A^{\prime} B^{\prime} D^{\prime}$ du mécanisme $M I$ sont interceptés par deux surfaces coniques de sommet $A$ et $A^{\prime}$, de demi-angle au sommet $\phi$, dont les axes sont colinéaires au vecteur vitesse $V_{1}$ du bloc. Ces surfaces coniques sont 
tangentes i) au plan de base suivant les génératrices $A C$ et $A^{\prime} C^{\prime}$ et ii) aux plans latéraux $A B D$ et $A^{\prime} B^{\prime} D^{\prime}$ suivant les génératrices $A M$ et $A^{\prime} M^{\prime}$. A la surface du sol, la directrice suit un arc d'ellipse $M C$ (respectivement $M^{\prime} C^{\prime}$ ) tangente i) à la trace du plan latéral au point $M$ (respectivement $M^{\prime}$ ) et ii) à la trace du plan de base au point $C$ (respectivement $C^{\prime}$ ). Le mécanisme de rupture ainsi défini respecte la condition cinématique : toutes les surfaces en rupture sont inclinées d'un angle $\phi$ par rapport au vecteur vitesse $V_{l}$. Comme pour le mécanisme $M l$, ce mécanisme est défini par le seul paramètre angulaire $\beta_{l}$, l'angle dièdre entre le plan horizontal et le plan de base $A A^{\prime} C C^{\prime}$. La cinématique du mécanisme $M l t$ est similaire à celle du mécanisme $M I$.

\section{Résultats numériques}

La méthode cinématique de la théorie de l'analyse limite requiert le calcul de la puissance des efforts extérieurs et de la puissance dissipée pour chaque mécanisme envisagé. La puissance des efforts extérieurs concerne la force de butée $P_{P}$ et le poids des différents blocs. Au sein des mécanismes de rupture, il y a dissipation d'énergie suivant les surfaces de discontinuité de vitesses. L'écriture de l'équation du bilan énergétique pour les différents mécanismes permet de donner l'expression de l'effort de butée $P_{P}$ sous la forme :

$$
P_{P}=K_{p \gamma} \frac{h^{2} b}{2}
$$

où $K_{p \gamma}$ représente le coefficient de butée tridimensionnelle. Ce coefficient est fonction des différents paramètres angulaires décrivant chacun des mécanismes envisagés, de l'angle de frottement interne du sol $\phi$, de l'angle de frottement solstructure $\delta$ et du rapport largeur sur hauteur de l'écran $b / h$.

Pour chaque mécanisme, un code informatique spécifique a été élaboré. Ces codes exigent en entrée les caractéristiques géométriques de l'écran ( $b$ et $h$ ) et les caractéristiques mécaniques du sol et de l'interface sol-écran ( $\phi$ et $\delta$ ). La minimisation du coefficient de butée $K_{p \gamma}$ par rapport aux paramètres angulaires du mécanisme considéré permet d'obtenir le coefficient de butée minimal ainsi que le volume critique correspondant. Cette minimisation a été effectuée numériquement en utilisant la méthode du gradient conjugué.

Dans les paragraphes suivants, on présente tout d'abord les résultats obtenus pour chacun des trois mécanismes de rupture envisagés. La confrontation des résultats entre mécanismes permet, ensuite, de dégager le meilleur majorant de l'effort de butée.

Nous rappelons que, suite à l'analyse de nos résultats expérimentaux, nous avons limité cette étude au domaine des écrans peu élancés $(b / h>0,25)$. En effet, les mécanismes envisagés ne prennent pas en compte le refoulement latéral du sol observé en partie inférieure de l'écran pour des élancements importants. 


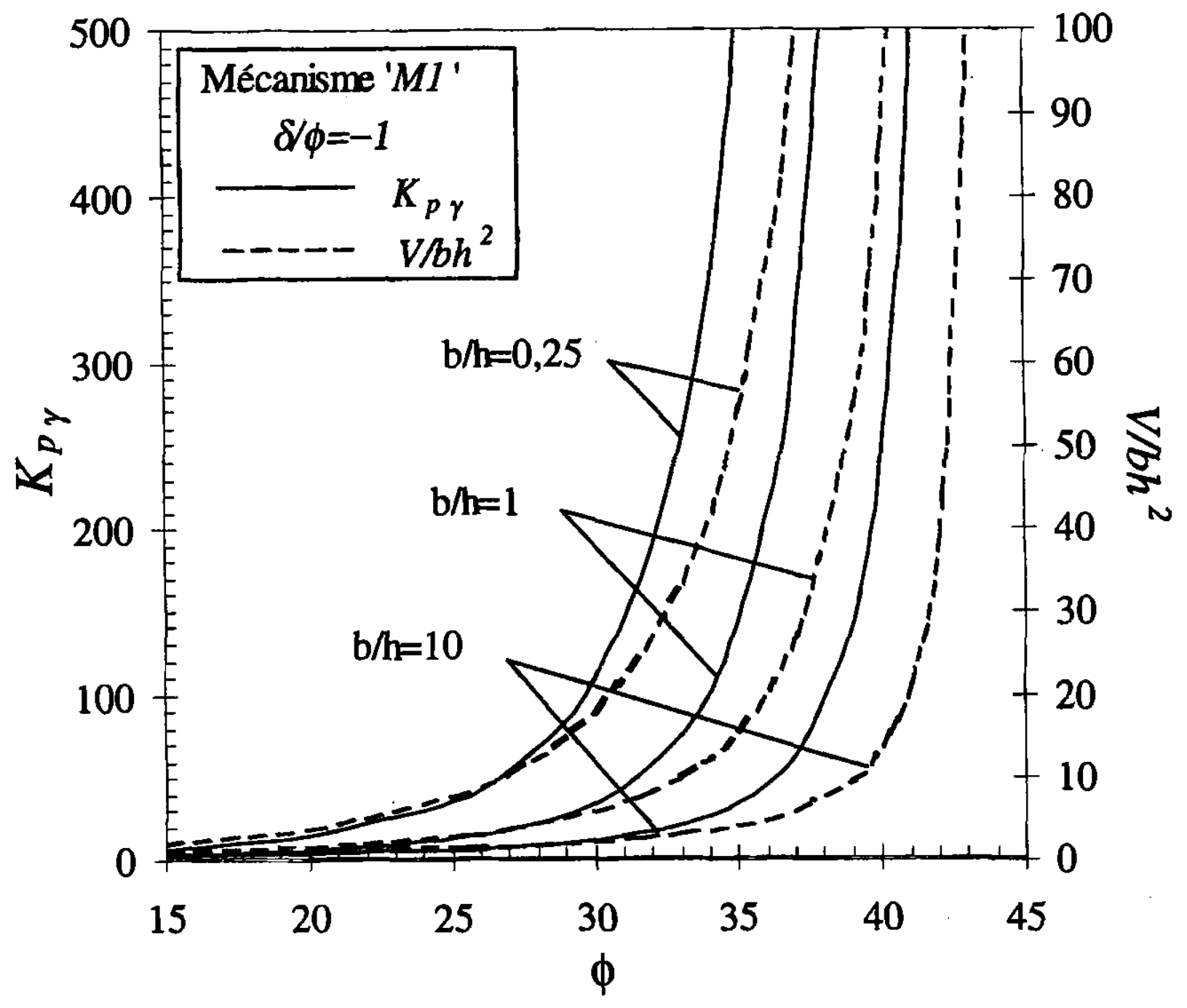

Figure 16. Variation de $K_{p \gamma}$ et du volume adimensionnel $V /(b h)^{2}$ en fonction de $\phi$ pour le mécanisme $M 1$. Variation of $\mathrm{K}_{\mathrm{py}}$ and $\mathrm{V} /(\mathrm{bh})^{2}$ with $\phi$ for the $\mathrm{Ml}$ mechanism

\subsection{Résultats du mécanisme M1}

Nous présentons sur la figure 16 l'évolution du coefficient de butée $K_{p \gamma}$ et du volume adimensionnel du bloc de sol en rupture $V /(b h)^{2}$ en fonction de l'angle de frottement interne du sol $\phi$ pour $\delta / \phi=-1$ et pour trois valeurs de $b / h(b / h=0,25 ; 1$ et 10).

Le coefficient de butée augmente logiquement avec $\phi$ et diminue avec l'accroissement du rapport $b / h$. Il est à remarquer que pour $\phi=45^{\circ}$, le coefficient $K_{p \gamma}$ tend vers l'infini. En effet, pour cette valeur de $\phi$, la condition restrictive concernant l'ouverture des plans latéraux (cf. équation [3]) conduit à prendre $\beta_{1}<0$ et, dans ce cas, le bloc de sol en rupture ne débouche plus en surface.

La figure 17 illustre l'évolution de la surface débouchante en fonction de $\phi\left(\phi=20^{\circ}\right.$, $30^{\circ}$ et $40^{\circ}$ ) pour $\delta / \phi=-1$ et $b / h=1$. 


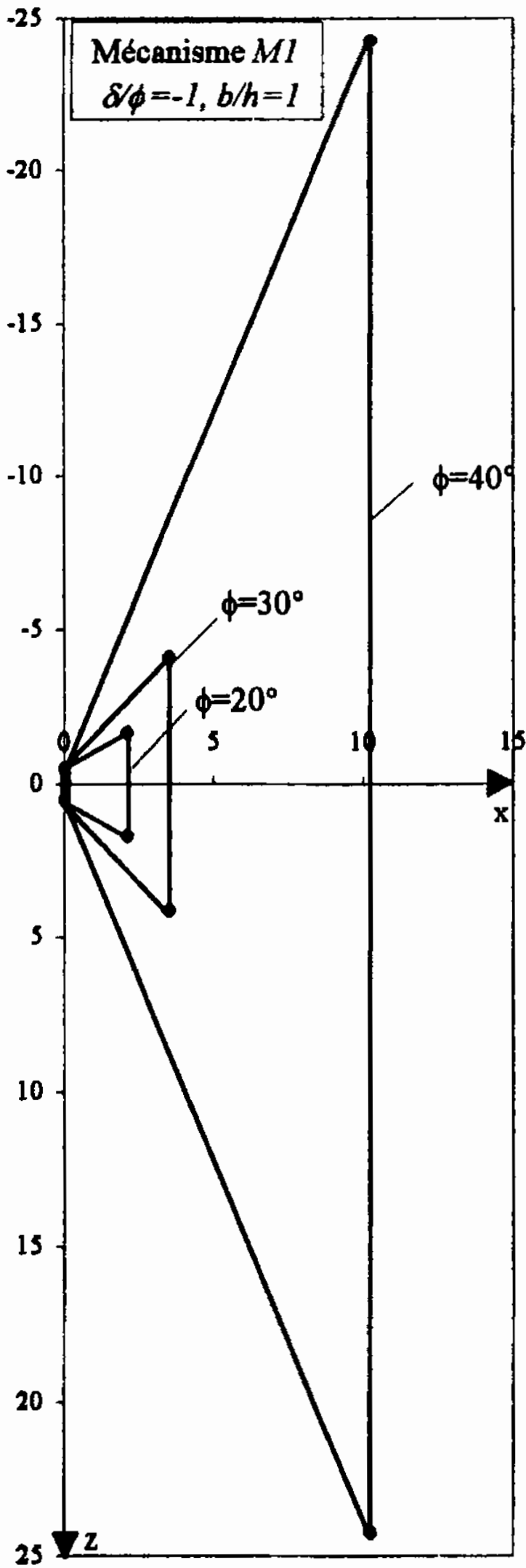

Figure 17. Surfaces débouchantes du mécanisme $M I$ pour $\phi=20^{\circ}, 30^{\circ}$ et $40^{\circ}$; $\delta / \phi=-1$ avec $b / h=1$. Plan view of the M1 mechanism for $\phi=20^{\circ}, 30^{\circ}$ and $40^{\circ}$; $\delta / \phi=-1$ and $b / h=1$

La figure 17 montre clairement que les plans latéraux s'épanouissent avec l'augmentation de $\phi$ pour tendre vers le plan de l'écran. Le mécanisme $M 1$ mobilise un volume de sol très important pour les valeurs moyennes à fortes de l'angle de frottement $\phi$ et conduit ainsi à une surestimation du coefficient de butée. D'autre 
part, ce mécanisme contredit les observations qualitatives de Meksaouine [MEK 93] qui ont montré que la surface débouchante du mécanisme est délimitée par une ligne courbe ne présentant pas de points anguleux (cf. figure 1).

\subsection{Résultats du mécanisme M2}

Comme dans le cas des écrans filants, le mécanisme présent permet d'améliorer la solution issue du mécanisme $M 1$. Nous présentons sur la figure 18 l'évolution du coefficient de butée $K_{p \gamma}$ et du volume adimensionnel $V /(b h)^{2}$ en fonction de l'angle de frottement interne du sol $\phi$ pour $\delta / \phi=-1$ et pour trois valeurs de $b / h(b / h=0,25$; 1 et 10$)$.

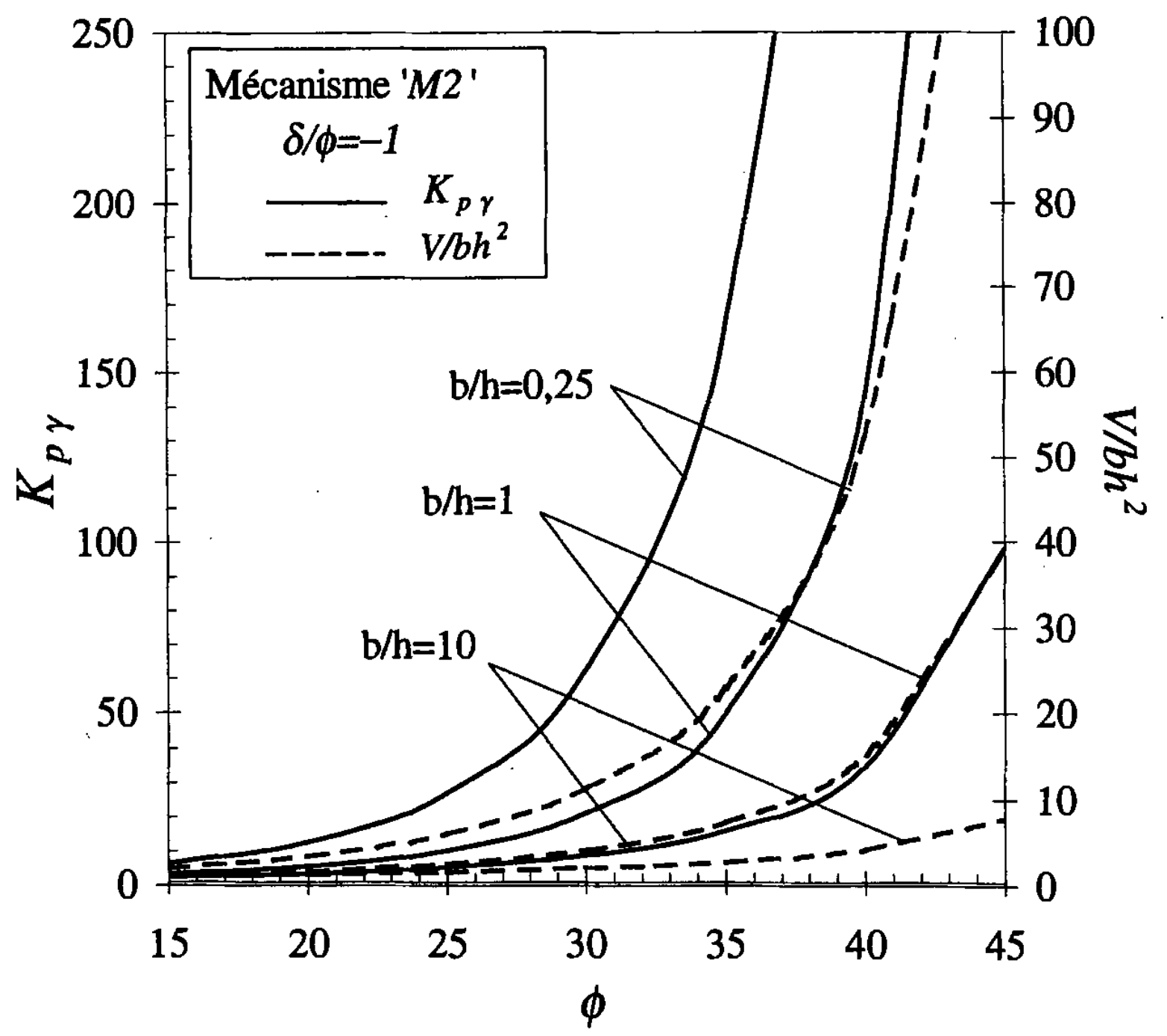

Figure 18. Variation de $K_{p \gamma}$ et du volume adimensionnel $V /(b h)^{2}$ en fonction de $\phi$ pour le mécanisme $M 2$. Variation of $\mathrm{K}_{\mathrm{py}}$ and $\mathrm{V} /(\mathrm{bh})^{2}$ with $\phi$ for the $\mathrm{M} 2$ mechanism

Contrairement au cas du monobloc, le coefficient $K_{p \gamma}$ est défini pour $\phi=45^{\circ}$. Ce mécanisme offre une flexibilité plus importante, la surface de base bilinéaire représentant de manière plus réaliste la surface expérimentale incurvée pour les fortes valeurs de $\phi$ et de $\delta$. 
La figure 19 illustre l'évolution de la surface débouchante en fonction de $\phi\left(\phi=20^{\circ}\right.$, 30 et $40^{\circ}$ ) pour $\delta / \phi=-1$ et $b / h=1$.

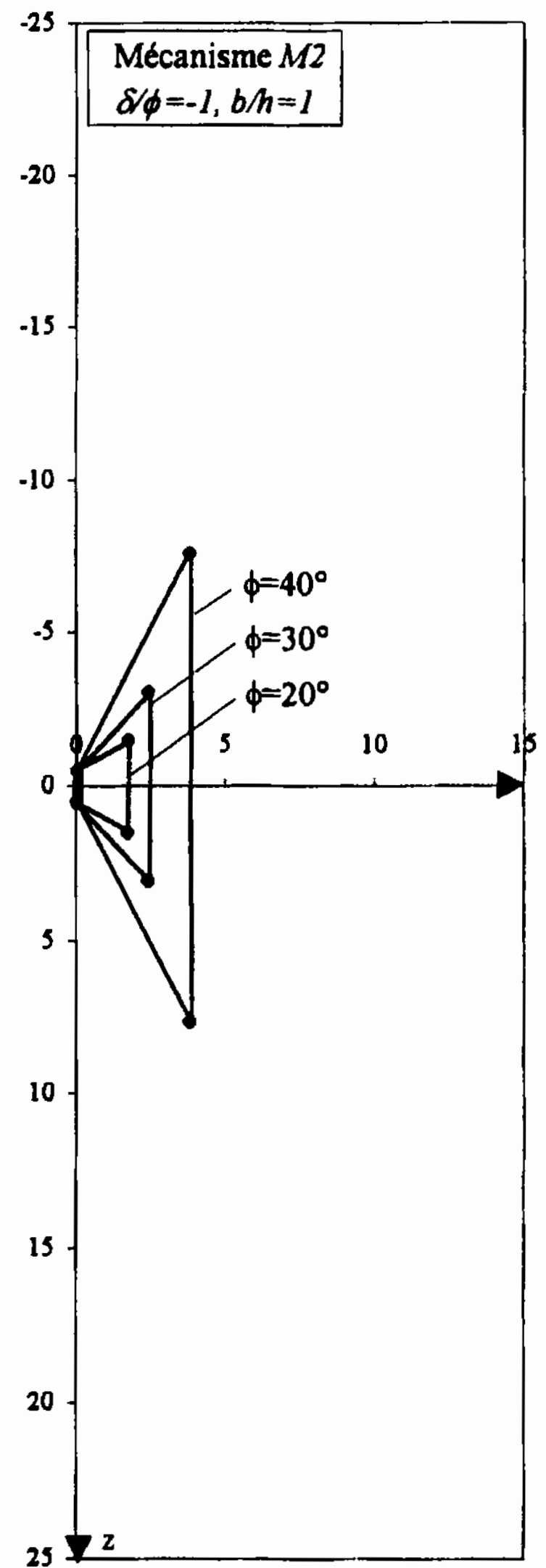

Figure 19. Surfaces débouchantes du mécanisme $M 2$ pour $\phi=20^{\circ}$, 30 et $40^{\circ}$; $\delta / \phi=-1$ avec $b / h=1$. Plan view of the M2 mechanism for $\phi=20^{\circ}, 30^{\circ}$ and $40^{\circ}$; $\delta / \phi=-1$ and $b / h=1$ 
L'aire de la surface débouchante du mécanisme $M 2$ est fortement réduite par rapport à celle donnée par le mécanisme $M 1$. Cette réduction est d'autant plus significative que la valeur de $\phi$ est importante (cf. figures 17 et 19). Cependant, comme pour le mécanisme $M 1$, la surface débouchante présente des points anguleux, ce qui est contraire aux observations sur modèles.

\subsection{Résultats du mécanisme M1t :}

Nous présentons sur la figure 20 l'évolution du coefficient de butée $K_{p \gamma}$ et du volume adimensionnel $V /(b h)^{2}$ en fonction de l'angle de frottement interne du sol $\phi$ pour $\delta / \phi=-1$ et pour trois valeurs de $b / h(b / h=0,25 ; 1$ et 10$)$.

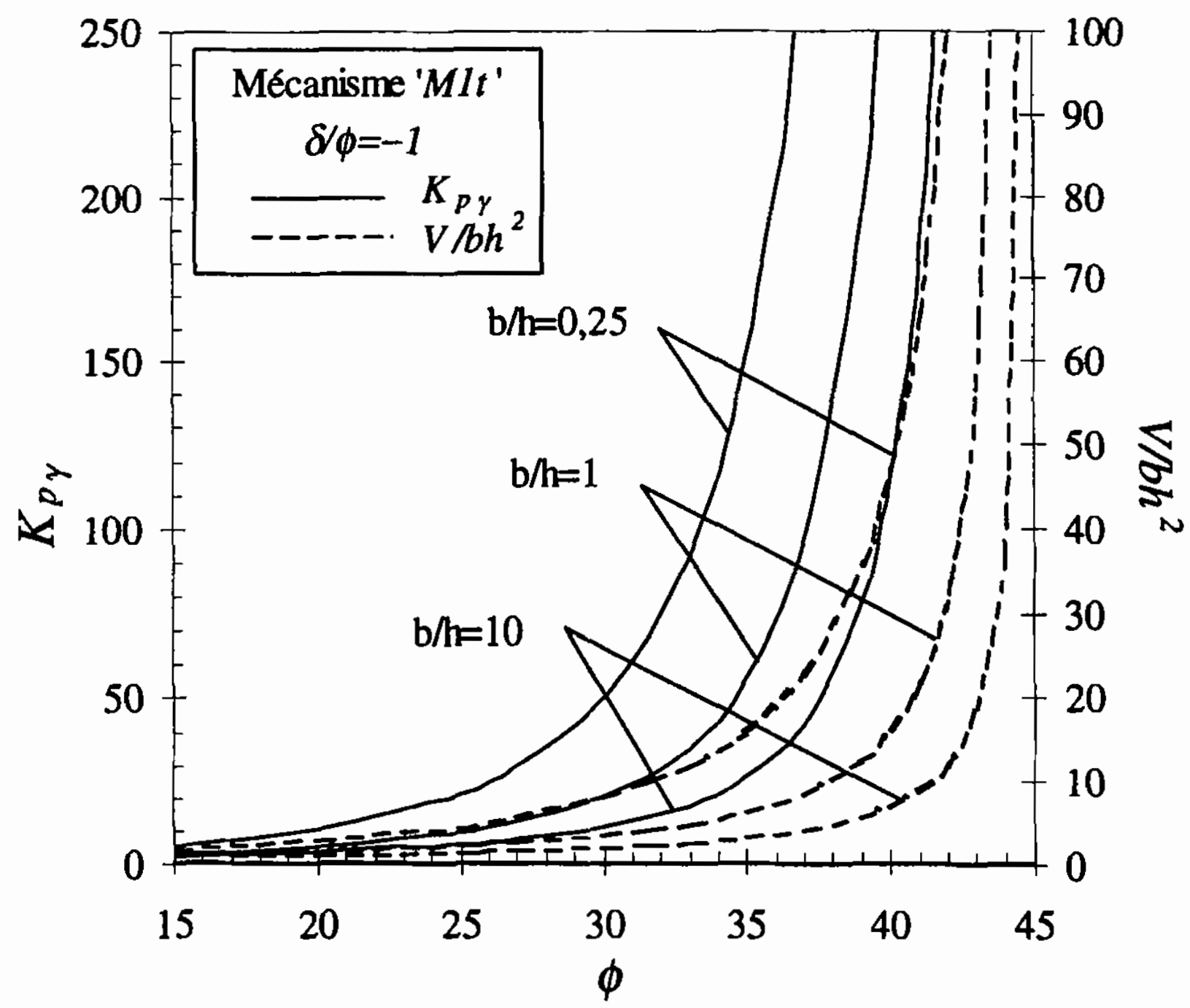

Figure 20. Variation de $K_{p \gamma}$ et du volume adimensionnel $V /(b h)^{2}$ en fonction de $\phi d u$ mécanisme M1t. Variation of $\mathrm{K}_{\mathrm{pr}}$ and $\mathrm{V} /(\mathrm{bh})^{2}$ with $\phi$ for the M1t mechanism

Comme pour le mécanisme $M I$, le mécanisme $M I t$ conduit à une valeur infinie du coefficient de butée pour $\phi=45^{\circ}$. En effet, ce mécanisme ne présente pas suffisamment de liberté pour simuler la rupture dans le cas d'un sol dense et d'un écran rugueux. 
L'évolution de la surface débouchante en fonction de $\phi\left(\phi=20^{\circ}, 30^{\circ}\right.$ et $\left.40^{\circ}\right)$ pour $\delta / \phi=-1$ et $b / h=1$ est représentée sur la figure 21 .

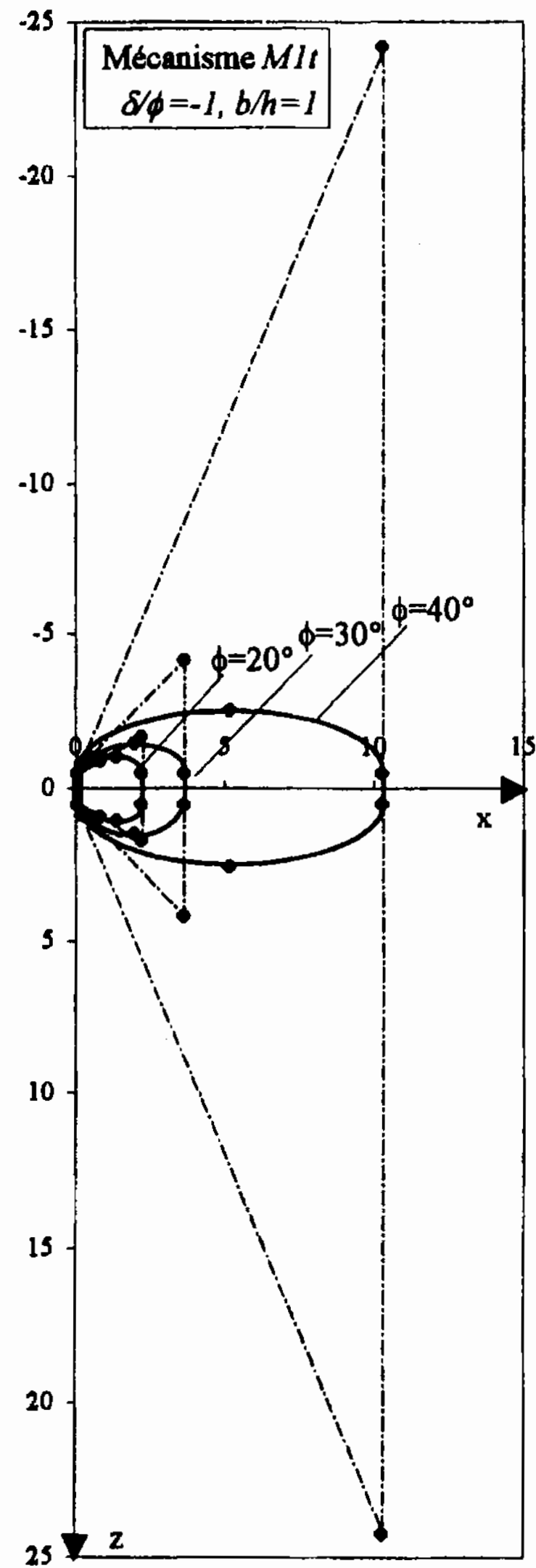

Figure 21. Surfaces débouchantes du mécanisme MIt pour $\phi=20^{\circ}, 30^{\circ}$ et $40^{\circ}$; $\delta / \phi=-1$ avec $b / h=1$. Plan view of the M1t mechanism for $\phi=20^{\circ}, 30^{\circ}$ and $40^{\circ}$; $\delta / \phi=-1$ and $b / h=1$ 
Au fur et à mesure de l'augmentation de $\phi$, les plans latéraux s'estompent et laissent place aux surfaces coniques de raccordement. On peut également noter l'augmentation importante de la surface débouchante entre $30^{\circ}$ et $40^{\circ}$, le mécanisme présent n'étant pas adapté aux fortes valeurs de $\phi$ et de $\delta$.

\section{Confrontation entre mécanismes}

La solution obtenue à partir du mécanisme $M I$ fournit toujours le plus grand majorant parmi les trois mécanismes étudiés. Dans la suite de notre étude, on se limitera à la confrontation entre les mécanismes $M 2$ et $M 1 t$.
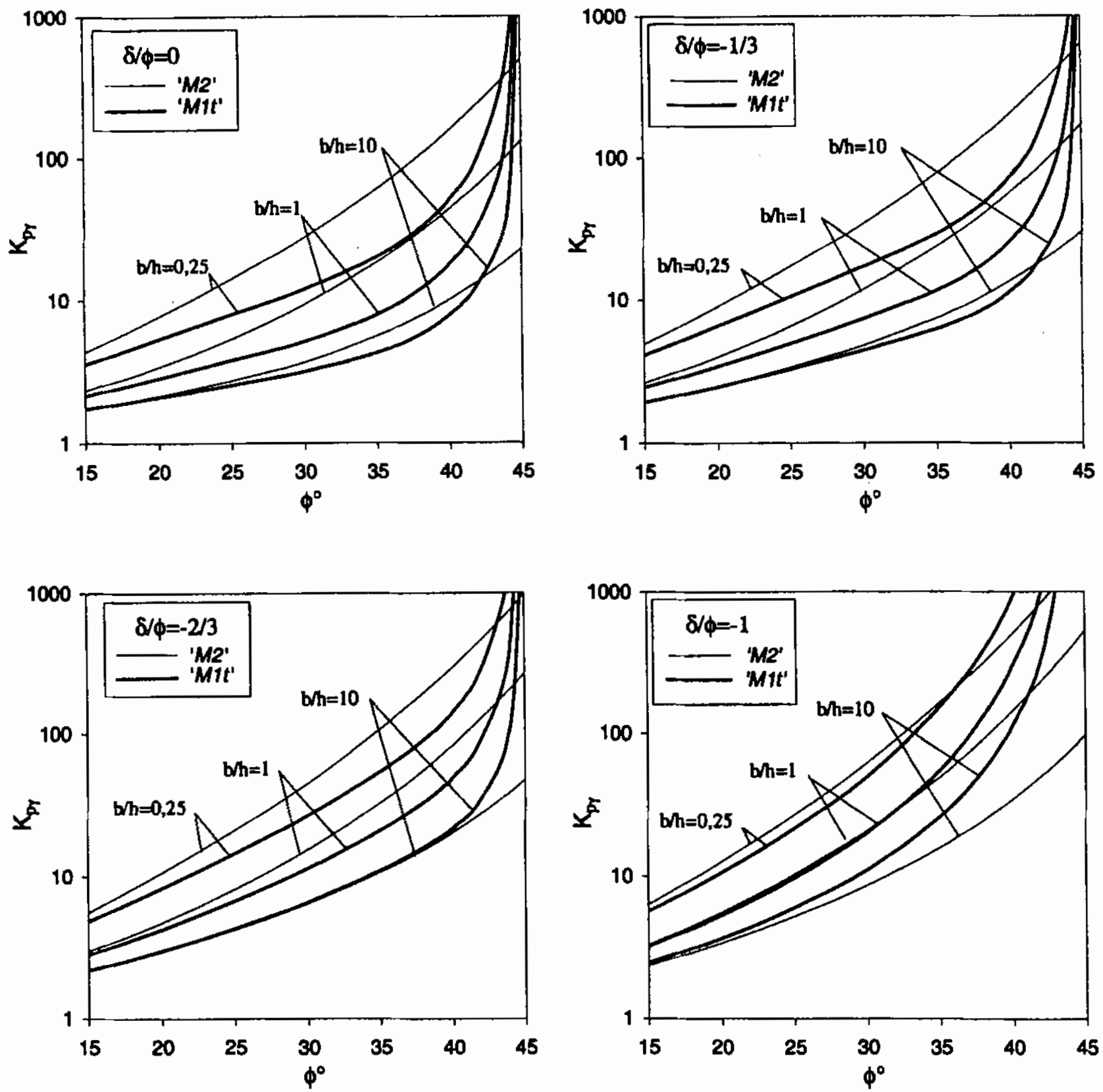

Figure 22. Variation de $K_{p y}$ en fonction de $\phi$ pour $b / h=0,25 ; 1$ et 10 et pour $\delta / \phi=0 ;-1 / 3 ;-2 / 3$ et -1 . $\mathrm{K}_{\mathrm{py}}$ versus $\phi$ for $\mathrm{b} / \mathrm{h}=0.25 ; 1$ and 10 and for $\delta / \phi=0$; $-1 / 3 ;-2 / 3$ and -1 
La figure 22 présente la variation du coefficient de butée $K_{p \gamma}$ issu des mécanismes $M 2$ et $M I t$ en fonction de $\phi$ pour trois valeurs du rapport $b / h(b / h=0,25 ; 1$ et 10$)$ et pour $\delta / \phi=0 ;-1 / 3 ;-2 / 3$ et -1 .

Pour les écrans lisses à moyennement rugueux $(\delta / \phi>-2 / 3)$, le mécanisme $M 1 t$ aboutit au meilleur majorant de l'effort de butée sauf pour les fortes valeurs de l'angle de frottement interne du sol $\phi$. L'amélioration amenée par le mécanisme $M 1 t$ est d'autant plus significative que le rapport $b / h$ diminue. Par contre, pour les écrans rugueux $(\delta / \phi=-1)$, le mécanisme $M 2$ supplante le mécanisme $M 1 t$ dans le cas des sols denses et/ou dans le cas des écrans d'élancement faible.

Le mécanisme aboutissant au meilleur majorant n'étant pas unique, on présente dans le tableau 1 le plus faible majorant issu des mécanismes $M 2$ et $M I t$ pour différentes configurations géométriques et mécaniques.

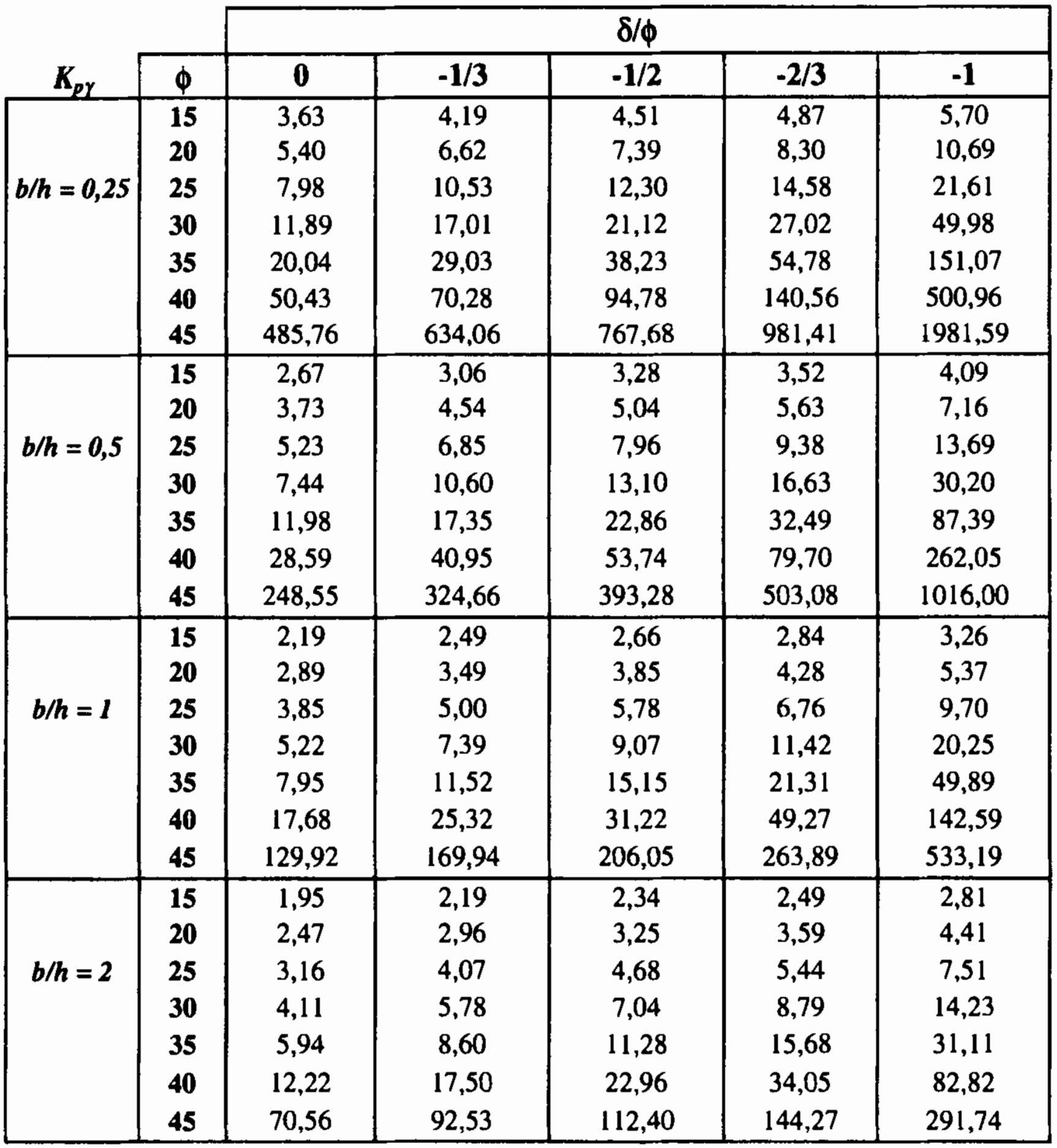




\begin{tabular}{|c|c|c|c|c|c|c|}
\hline \multirow[b]{2}{*}{$\boldsymbol{K}_{p \gamma}$} & & \multicolumn{5}{|c|}{$\delta / \phi$} \\
\hline & $\phi$ & $\mathbf{0}$ & $-1 / 3$ & $-1 / 2$ & $-2 / 3$ & -1 \\
\hline \multirow{7}{*}{$b / h=5$} & 15 & 1,80 & 2,02 & 2,15 & 2,28 & 2,49 \\
\hline & 20 & 2,21 & 2,63 & 2,89 & 3,17 & 3,68 \\
\hline & 25 & 2,74 & 3,51 & 4,01 & 4,63 & 5,82 \\
\hline & 30 & 3,44 & 4,80 & 5,81 & 7,19 & 10,07 \\
\hline & 35 & 4,73 & 6,85 & 8,94 & 12,27 & 19,81 \\
\hline & 40 & 8,94 & 12,81 & 16,81 & 24,93 & 46,91 \\
\hline & 45 & 34,82 & 45,96 & 56,09 & 72,41 & 146,78 \\
\hline \multirow{7}{*}{$b / h=10$} & 15 & 1,75 & 1,96 & 2,08 & 2,18 & 2,38 \\
\hline & 20 & 2,13 & 2,52 & 2,76 & 2,98 & 3,43 \\
\hline & 25 & 2,60 & 3,32 & 3,78 & 4,27 & 5,25 \\
\hline & 30 & 3,22 & 4,47 & 5,39 & 6,48 & 8,67 \\
\hline & 35 & 4,33 & 6,27 & 8,15 & 10,74 & 16,01 \\
\hline & 40 & 7,85 & 11,24 & 14,75 & 20,39 & 34,89 \\
\hline & 45 & 22,76 & 30,28 & 37,18 & 48,37 & 98,35 \\
\hline \multirow{7}{*}{$\begin{array}{c}\text { filant } \\
\text { (2 blocs) }\end{array}$} & 15 & 1,70 & 1,89 & 1,99 & 2,08 & 2,27 \\
\hline & 20 & 2,04 & 2,39 & 2,58 & 2,78 & 3,18 \\
\hline & 25 & 2,46 & 3,08 & 3,44 & 3,82 & 4,66 \\
\hline & 30 & 3,00 & 4,06 & 4,71 & 5,46 & 7,24 \\
\hline & 35 & 3,69 & 5,49 & 6,73 & 8,22 & 12,16 \\
\hline & 40 & 4,60 & 7,73 & 10,11 & 13,27 & 22,71 \\
\hline & 45 & 5,83 & 11,42 & 16,34 & 23,56 & 49,42 \\
\hline
\end{tabular}

Tableau 1. Valeurs du coefficient de butée $K_{p \gamma}$ pour différentes configurations mécaniques et géométriques. Values of passive earth pressure coefficient $\mathrm{K}_{\mathrm{pr}}$ for different mechanical and geometrical configurations

\section{Méthode pratique d'évaluation de la butée tridimensionnelle}

Les essais effectués par [BEL 88] et [MEK 93] ont mis en évidence que le fuseau de points de mesure vérifiant l'équation [2] correspond à un facteur de forme $C$ compris entre 0,4 et 0,8 pour $b / h>0,25$ dans le cas du sable d'Hostun (figure 8).

Le calcul du facteur de forme à partir de nos résultats théoriques montre que ce coefficient n'est pas constant dans la plage des valeurs présentées dans le tableau 1. Il dépend à la fois de l'angle de frottement du sol $\phi$ et de la rugosité de l'écran $\delta$. Néanmoins, pour des angles de frottement $\phi$ faibles à modérés $\left(\phi<35^{\circ}\right)$ et dans le cas d'écrans lisses $(-1 / 2<\delta / \phi<0)$, ce coefficient est peu sensible à la variation de $\delta$. Dans ce domaine de valeurs de $\phi$ et de $\delta$, nous proposons une méthode pratique d'évaluation de la butée tridimensionnelle basée sur l'équation [2]. 
Les valeurs numériques du facteur de forme $C$ obtenues par notre modèle théorique peuvent être approchées par une expression simple fonction seulement de l'angle de frottement :

$$
C=2,50 \operatorname{tg}^{2} \phi-0,48 \operatorname{tg} \phi+0,27
$$

L'écart maximal entre la valeur issue de la formule simplifiée [5] et celle obtenue par notre approche théorique est dans tous les cas inférieur à $9 \%$ avec un écart moyen d'environ $3 \%$.

Pour les essais de Meksaouine [MEK 93], l'application de cette relation conduit à une valeur $C=1,12$ pour $\phi=34,5^{\circ}$, alors que l'enveloppe des résultats expérimentaux correspond à un facteur de forme compris entre 0,4 et 0,8 . Cet écart confirme que les schémas proposés, qui constituent la meilleure borne supérieure existante, surestiment la butée mobilisée et méritent d'être améliorés.

\section{Conclusion}

L'étude expérimentale de la butée tridimensionnelle a été menée sur un modèle réduit non centrifugé allant des écrans filants à ceux d'élancement important. Cette étude a permis de mettre en évidence deux types de comportement bien distincts. L'un pour les écrans peu élancés $(h / b<4)$ pour lequel le prisme de rupture tridimensionnel prend naissance à la base de l'écran et l'autre pour un élancement important pour lequel le prisme de rupture tridimensionnel ne concerne que lá vartie supérieure de l'écran associé à un refoulement latéral du sol dans sa partie inférieure. L'étude théorique a porté sur les écrans de faible élancement. Trois mécanismes de rupture tridimensionnels constitués d'un ou deux blocs rigides ont été envisagés dans le cadre de l'approche cinématique de l'analyse limite. La confrontation avec les résultats expérimentaux de Meksaouine [MEK 93] montre que les mécanismes finalement retenus sont satisfaisants vis-à-vis de la forme du volume de sol en rupture. Les modèles théoriques méritent néanmoins d'être améliorés car, bien que constituant la meilleure borne supérieure existante, ils surestiment encore les valeurs expérimentales de l'effort de butée. L'ensemble des résultats expérimentaux et théoriques a conduit à proposer une formulation simplifiée de la butée d'un sol frottant sur un écran de faible élancement à partir de

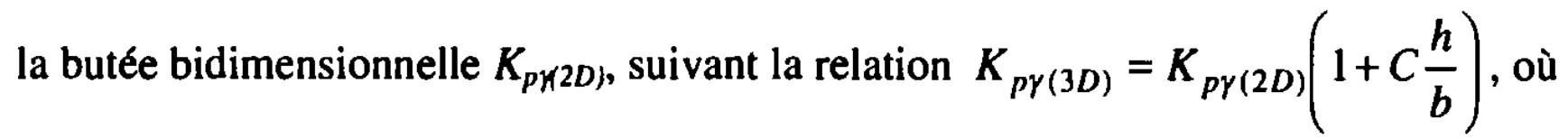
le facteur de forme $\mathrm{C}$ est fonction de l'angle de frottement interne du sol. 


\section{Bibliographie}

[ABD 94] ABDI R., DE BUHAN P., PASTOR J., « Calculation of the critical height of a homogenized reinforced soil wall: a numerical approach », International Journal for Numerical and Analytical Methods in Geomechanics, Vol. 18, p. 485-505, 1994.

[ABD 97] ABDi R., ShaHROUR I., «Etude de la stabilité des murs en terre armée sous chargements sismiques ", Revue Française de Génie Civil, Vol. 1, n³, p. 569-584, 1997.

[BAS 79] BASUdhaR P. K., SINGH D. N., " A generalized procedure for predicting optimal lower bound break-out factors of strip anchors ", Géotechnique, Vol. 44, $n^{\circ} 2$, p. 307-318, 1979.

[BEL 88] Belabdelouhab F., Etude expérimentale de la butée discontinue sur modèle réduit, Thèse de Doctorat, 214 p., INSA Lyon, 1988.

[BLU 32] BLUM H., "Wirtschaftliche Dalbenformen und deren Berechnung ", Bautechnik, Vol. 10, n 5, p. 122-135, 1932.

[BOU 77] BourdeaU Y., Poussée des sols pulvérulents, influence des caractéristiques des sols et du modèle d'essais, Thèse de Docteur Ingénieur, 205 p., INSA Lyon, 1977.

[BR1 66] BRINCH HANSEN J., "Resistance of a rectangular slab", Danish Geotechnisk Institut Bulletin, Vol. 21, n 5, p. 12-24, 1966.

[BUC 30] BuCHHOLZ W., "Erdwiderstand auf Ankerplatten", Jahrbuch der Hafenbautechnischen Gesellschaft, n ${ }^{\circ}$ 123, 300 p., 1930.

[CHE 70] CHEN W.F., SCAWTHORN C. R., « Limit analysis and limit equilibrium solutions in soil mechanics ", Soils and Foundations, Vol. 10, n 3, p. 13-49, 1970.

[CHE 73] CHEN W.F., ROSENFARB J.L., « Limit analysis solutions of earth pressure problems ", Soils and Foundations, Vol. 13, n 4, p. 45-60, 1973.

[CHE 75] CHEN W.F., « Limit analysis and soil plasticity », Amsterdam, Elsevier, 637 p., 1975.

[COL 69] CollinS I.F., "The upper-bound theorem for rigid/plastic solids to include Coulomb friction ", J. Mech. Phys. Solids, Vol. 17, p. 323-338, 1969.

[COL 73] COLLINS I.F., "A note on the interpretation of Coulomb's analysis of the thrust on a rough retaining wall in terms of the limit theorems of plasticity theory ", Géotechnique, Vol. 24, n 1, p. 106-108, 1973.

[COR 86] CORTE J. F., GARNIER J., "Une centrifugeuse pour la recherche en géotechnique ", Bulletin de liaison L.P.C., $\mathrm{n}^{\circ}$ 146, p. 5-28, 1986. 
[DAV 79] DAvis A., AugER D., "La butée des sables : essais en vrai grandeur », Annales de l'Institut Technique du Bâtiment et des Travaux Publics, $\mathrm{n}^{\circ} 375,24$ p., 1979.

[DEB 93] DE BUHAN P., SALENÇON J., * A comprehensive stability analysis of soil nailed structures ", Eur. J. Mech., A/Solids, Vol. 12, n 3, p. 325-345, 1993.

[DEB 98] DE BUHAN P., GARNIER D., « Three dimensional bearing capacity analysis of a foundation near a slope ", Soils and Foundations, Vol. 38, n ${ }^{\circ} 3$, p. 153-163, 1998.

[DRE 93] DRESCHER A., DETOURNAY E., " Limit load in translational failure mechanisms for associative and non-associative materials", Géotechnique, Vol. 43, n 3, p. 443-456, 1993.

[DRU 52] DRUCKeR D. C., Prager W., "Soil mechanics and plastic analysis or limit design », Q. Appl. Math., Vol. 10, p. 157-165, 1952.

[FIN 67] FINN W. D. L., « Applications of limit plasticity in soil mechanics », J. Soil Mechanics and Foundation Division, ASCE, Vol. 93, SM5, p. 101-119, 1967.

[HER 85] HeRITIER B., Plumelle C., « Mobilisation progressive de la butée dans les sables ", Proc. $11^{\text {th }}$ Int. Conf. on Soils Mechanics and Foundation Engng., San Francisco, Vol. 4, p. 2091-2094, 1985.

[HOR 67] HORN A., « Raümlicher Erwiderstand in nicht Bindingen Böden », V.O.I. Zeitschrift, ${ }^{\circ} 109$, p. 585-615, 1967.

[HOR 72] HORN A., "Résistance et déplacement de culées de ponts chargées latéralement ", $5^{\text {th }}$ European Conference on Soil Mechanics and Foundation Engineering, Madrid, Vol. 2, p. 143-145, 1972.

[HUE 57] HUUECKEL S., « Model tests on anchoring capacity of vertical and inclined plates", Proc. Fourth Int. Conference on Soil Mechanics and Foundation Engineering, Vol.2, p. 203-206, Londres, 1957.

[KER 72] KERISEL J., "Le langage des modèles en mécanique des sols ", $5^{\text {th }}$ European Conference on Soil Mechanics and Foundation Engineering, Madrid, Vol. 2, p. 9-30, 1972.

[KER 90] KERISEL J., ABSI E., Tables de butée et de poussée, $3^{\mathrm{e}}$ édition, Presses de l'ENPC 1990.

[LEE 72] LEE I.K., HERINGTON J.R., «A theoretical study of the pressures acting on a rigid walls by a sloping earth on rockfill », Géotechnique, Vol. 22, $\mathrm{n}^{\circ} 1$, p. 1-26, 1972.

[LYS 70] LYSMER J., « Limit analysis of plane problems in soil mechanics », J. Soil Mech. and Found. Div., ASCE, Vol. 96, n' ${ }^{\circ}$ SM4, p. 1311-1334, 1970.

[MEK 93] MEKSAOUINE M., Etude expérimentale et théorique de la poussée passive sur pieux rigides, Thèse de Doctorat, 193 p., INSA Lyon, 1993. 
[MIC 99] MiCHaLOWSKI R.L., Closure on «Stability of uniformly reinforced slopes ", J. Geotech. and Geoenv. Engrg., ASCE, Vol. 125, n 1, p. 81-86, 1999.

[MRO 69] MROZ Z., DRESCHER A., « Limit plasticity approach to some cases of flow of bulk solids », J. Engng. Ind. Trans. Am. Soc. Mech. Engrs., n 91, p. 357-364, 1969.

[PAS 78] PASTOR J., «Analyse limite: Détermination de solutions statiques complètes. Application au talus vertical ", Journal de Mécanique Appliquée, Vol 2, $n^{\circ} 2$, p. 167-197, 1978.

[PAS 82] PASTOR J., TURGEMAN S., «Limit analysis in axisymmetrical problems : numerical determination of complete statical solutions », Int. J. of Mech. Sci., Vol. 24, $\mathrm{n}^{\circ} 2,95-117,1982$.

[PET 33] PetermanN H., «Bewegungen und Kraft bei Ankerplatten ", Bauingenieur, Vol. 14, 1933.

[REG 99] REGENASS P., Application de la méthode cinématique de l'analyse limite au calcul de la butée tridimensionnelle et de la charge limite de plaques d'ancrage superficielles, Thèse de Doctorat, 267 p., ULP Strasbourg, 1999.

[ROW 65] ROWE P.W., PEAKER K., «Passive earth pressure measurements", Géotechnique, Vol. 15, $\mathrm{n}^{\circ}$ 1, p. 57-78, 1965.

[SAL 83] SAlENÇON J., Calcul à la rupture et analyse limite, Presse de l'ENPC, Paris, 366 p., 1983.

[SAL 90] SALENÇON J., "An introduction to the yield design theory and its application to soil mechanics ", Eur. J. Mech., A/Solids, Vol 9, n 5, p. 477-500, 1990.

[SAL 93] SALENÇON J., "Yield design: a survey of the theory, In evaluation of the global bearing capacities of structures », ed. By G. Sacchi-Landriani, J. Salençon, Springer Verlag, Wien, New York, 1993.

[SOU 97] SOUBRA A.-H., "Seismic bearing capacity of shallow strip footings in seismic conditions", Geotechnical Engineering, The Institution of Civil Engineers, Vol. 125, $n^{\circ} 4$, p. 230-241, 1997.

[SOU 98a] Soubra A.-H., KASTNeR R., MASROURI F., Regenass P., «Etude de la stabilité d'ensemble des rideaux de soutènement ancrés ", Revue Française de Génie Civil, Editions Hermès, Vol. 2, n 4, p. 431-452, 1998.

[SOU 98b] Soubra A.-H., Kastner R., Benmansour A., « Etude de la butée des terres en présence d'écoulement ", Revue Française de Génie Civil, Editions Hermès, Vol. 2, nº 6, p. 691-707, 1998.

[SOU 99a] SOUBRA A.-H., "Upper-bound solutions for bearing capacity of foundations ", Journal of Geotechnical and Geoenvironmental Engineering, $A S C E$, Vol. 125, n 1, p. 59-68, 1999. 
[SOU 99b] Soubra A.-H., KaStNer R., BENMANSOUR A., " Passive earth pressures in the presence of hydraulic gradients ", Géotechnique, Vol. 49, n³, p. 319-330, 1999.

[WEI 61] WeISSENBACH A., "Der Erdwiderstand vor schmalen Druckflechen", Thèse de Docteur Ingénieur, Franzius Institut fur Grund und Wasserbau der Technischen Hochschule, Hannover, 338 p., 1961.

[ZWE 53] ZWECK H., «Erdwiderstand als raumliches Problem», Bautechnik, Vol. 7, $n^{\circ} 5$, p. 189-193, 1953. 